ARTICLE

\title{
Clostridioides difficile specific DNA adenine methyltransferase CamA squeezes and flips adenine out of DNA helix
}

Jujun Zhou (i) 1,3, John R. Horton (10) 1,3, Robert M. Blumenthal ${ }^{2}$, Xing Zhang ${ }^{1 凶} \&$ Xiaodong Cheng (i) ${ }^{1 凶}$

Clostridioides difficile infections are an urgent medical problem. The newly discovered C. difficile adenine methyltransferase $\underline{A}$ (CamA) is specified by all C. difficile genomes sequenced to date $(>300)$, but is rare among other bacteria. CamA is an orphan methyltransferase, unassociated with a restriction endonuclease. CamA-mediated methylation at CAAAAA is required for normal sporulation, biofilm formation, and intestinal colonization by C. difficile. We characterized CamA kinetic parameters, and determined its structure bound to DNA containing the recognition sequence. CamA contains an $\mathrm{N}$-terminal domain for catalyzing methyl transfer, and a C-terminal DNA recognition domain. Major and minor groove DNA contacts in the recognition site involve base-specific hydrogen bonds, van der Waals contacts and the Watson-Crick pairing of a rearranged A:T base pair. These provide sufficient sequence discrimination to ensure high specificity. Finally, the surprisingly weak binding of the methyl donor S-adenosyl-L-methionine (SAM) might provide avenues for inhibiting CamA activity using SAM analogs.

\footnotetext{
${ }^{1}$ Department of Epigenetics and Molecular Carcinogenesis, University of Texas MD Anderson Cancer Center, Houston, TX, USA. ${ }^{2}$ Department of Medica Microbiology and Immunology, and Program in Bioinformatics, The University of Toledo College of Medicine and Life Sciences, Toledo, OH, USA. ${ }^{3}$ These authors contributed equally: Jujun Zhou, John R. Horton. ${ }^{凶}$ email: XZhang21@mdanderson.org; XCheng5@mdanderson.org
} 
$\mathrm{T}$ here is an acute medical need to control gastrointestinal infections caused by Clostridioides difficile (also known as Clostridium difficile $)^{1-3}$. In the developed world, C. difficile is one of the leading causes of hospital-acquired infections, often following antibiotic therapy ${ }^{4}$. This pathogen produces endospores, making decontamination difficult ${ }^{5,6}$, and it causes deadly infections by producing significant toxins $\mathrm{s}^{7,8}$, and by stabilizing the colon bacterial population (microbiota, or microbiome) in an unhealthy distribution ${ }^{9,10}$. The US Centers for Disease Control and Prevention has classified C. difficile infections as being an urgent healthcare risk, associated with substantial morbidity and mortality ${ }^{11}$. Thus far, the medical need is unmet by therapeutic strategies in treatment of $C$. difficile infection and its recurrence ${ }^{12-15}$. Novel targeted therapeutics are urgently needed to combat $C$. difficile infection.

Post-synthetic methylations of DNA are common, and play significant roles, in a wide range of bacterial and archaeal cellular functions ${ }^{16-19}$, such as adenine methylation-directed mismatch repair in Escherichia coli by Dam ${ }^{20}$, and essential functions of the cell-cycle regulated adenine methyltransferase (MTase) CcrM in Caulobactor crescentus ${ }^{21}$. The majority of known bacterial and archaeal DNA MTases function to protect a cell's own DNA from digestion by a paired (cognate) restriction endonuclease ${ }^{22}$. However, there are bacterial "orphan" MTases (such as Dam and CcrM) - so named as they are not paired with a restriction endonuclease-that in many cases are involved in controlling chromosome replication, DNA repair, and gene expression ${ }^{16,23}$.

The newly discovered CamA enzyme (named for Clostridioides difficile adenine methyltransferase $\underline{\mathrm{A}}$ ) is another orphan MTase, is present in all C. difficile genomes sequenced to date $(>300)$, and is active in all $C$. difficile genomes subjected to PacBio singlemolecule real-time DNA sequencing (which can detect N6methyladenine-N6mA), but is rarely found in other bacteria ${ }^{24}$. CamA-mediated methylation at CAAAAA (underlining indicates the target $\mathrm{A}$ ) is required for normal sporulation and biofilm production by $C$. difficile, a key step in the disease transmission, as well as for colonization in animal models ${ }^{24}$. Here, we show the kinetic parameters and structural features of CamA in complex with cognate substrate DNA and, given the critical consequences of $C$. difficile infection to human health, discuss the potential for CamA as a novel therapeutic target.

\section{Results}

CamA exhibits weak binding of SAM. We purified recombinant full-length CamA [from gene CD2758 of reference strain $630^{25}$ ], and measured its enzymatic activity on a double stranded DNA oligonucleotide (oligo) containing a single recognition sequence (Fig. 1). We first optimized the reaction conditions for $\mathrm{pH}$, ionic strength, reaction time, and enzyme concentration (Supplementary Fig. 1). Under the optimal laboratory conditions $(\mathrm{pH} \mathrm{7.5,}$ $100 \mathrm{mM} \mathrm{NaCl}, t=2.5 \mathrm{~min}$ ), we next measured the CamA kinetic parameters by varying, respectively, concentrations of the DNA substrate and SAM (Fig. 1a, b). CamA has $k_{\text {cat }}$ values of $3.9 \mathrm{~min}^{-1}$ and $5.4 \mathrm{~min}^{-1}$, respectively, for substrate DNA and SAM. For comparison, the measured $k_{\text {cat }}$ values for the two other wellstudied DNA orphan adenine MTases, under their respective assay conditions, are $0.14 \mathrm{~min}^{-1}$ (E. coli Dam) ${ }^{26}$ and $5.2 \mathrm{~min}^{-1}$ (C. crescentus $\mathrm{CcrM})^{27}$. However, the CamA binding affinities (as reflected by $K_{\mathrm{m}}$ values) varied from $0.1 \mu \mathrm{M}$ for DNA to $>17 \mu \mathrm{M}$ for SAM (Fig. 1c). While the $K_{\mathrm{m}}$ value for DNA is comparable to that of E. coli Dam $(55 \mathrm{nM})^{26}$ and CcrM $(17-23 \mathrm{nM})^{27}$, CamA appears to have substantially lower affinity for the methyl donor SAM than that of E. coli Dam $(3-6 \mu \mathrm{M})^{26,28,29}$ and phage T4 Dam $(0.5 \mu \mathrm{M})^{30}$. For context, while we are not aware that SAM levels have been measured in C. difficile, in E. coli cells the concentration of SAM ranges from $\sim 30 \mu \mathrm{M}$ during logarithmic growth up to $\sim 230 \mu \mathrm{M}$ in stationary phase cells ${ }^{31}$.

To validate the weaker binding of SAM, we used isothermal titration calorimetry (ITC) to measure the dissociation constants $\left(K_{\mathrm{D}}\right)$ of CamA with SAM, the reaction product S-adenosylhomocysteine (SAH), and the SAM analog sinefungin (adenosylornithine) (Fig. 1d and Supplementary Fig. 2). Under constant conditions, CamA displayed the weakest binding to SAM $\left(K_{\mathrm{D}}=\right.$ 23-35 $\mu \mathrm{M})$, followed by sinefungin $(19 \mu \mathrm{M})$ and SAH $(8 \mu \mathrm{M})$. a

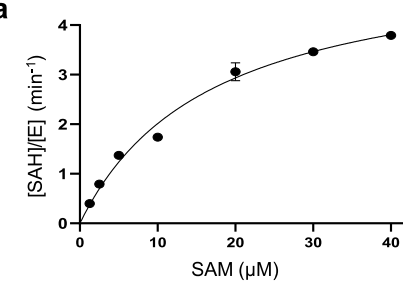

b

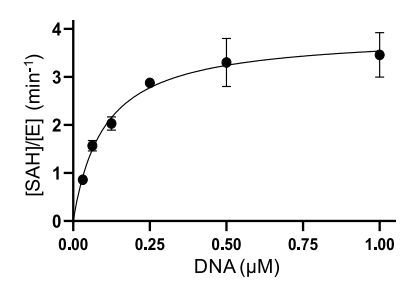

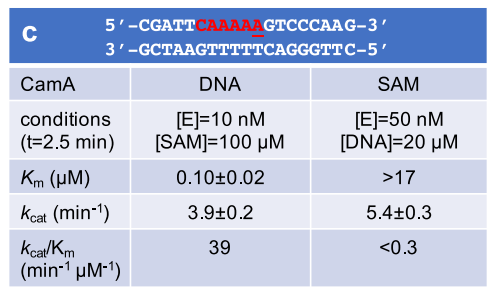

e

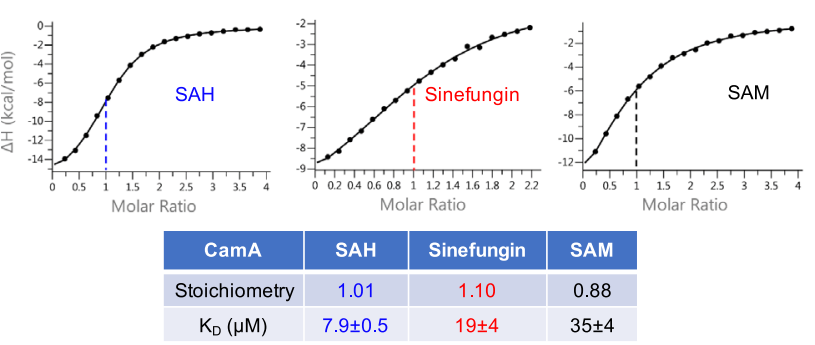

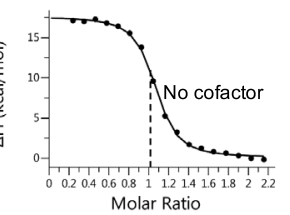
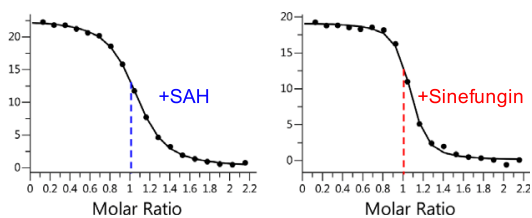

\begin{tabular}{|c|c|c|c|}
\hline CamA-DNA & No cofactor & SAH & Sinefungin \\
\hline Stoichiometry & 1.02 & 1.02 & 1.02 \\
\hline $\mathrm{K}_{\mathrm{D}}^{\mathrm{DNA}}(\mu \mathrm{M})$ & $0.20 \pm 0.02$ & $0.37 \pm 0.02$ & $0.11 \pm 0.02$ \\
\hline
\end{tabular}

Fig. 1 Activity of CamA. a, b The formation of byproduct SAH was measured in a bioluminescence assay, by varying concentrations of methyl donor SAM (a) or substrate DNA $(\mathbf{b})(N=2)$. The dependence of the velocity of product SAH formation per enzyme molecule [SAH]/[E] on substrate concentration was analyzed according to the Michaelis-Menten equation. c Summary of CamA kinetic parameters. The DNA substrate used is also shown. Data represent the mean \pm SD of two independent determinations, with duplicates assayed for each of the two determinations $(N=2)$. Source data are provided as a Source Data file. d ITC measurements of dissociation constants $\left(K_{D}\right)$ and stoichiometry of CamA for SAM, SAH, and sinefungin, with $N$ number of independent determinations ( $N=3$ for $\mathrm{SAH}, \mathrm{N}=2$ each for SAM and sinefungin) (Supplementary Fig. 2). e Influence of cofactor on CamA-DNA binding dissociation (two independent determinations $N=2$; Supplementary Fig. 3b, c). 

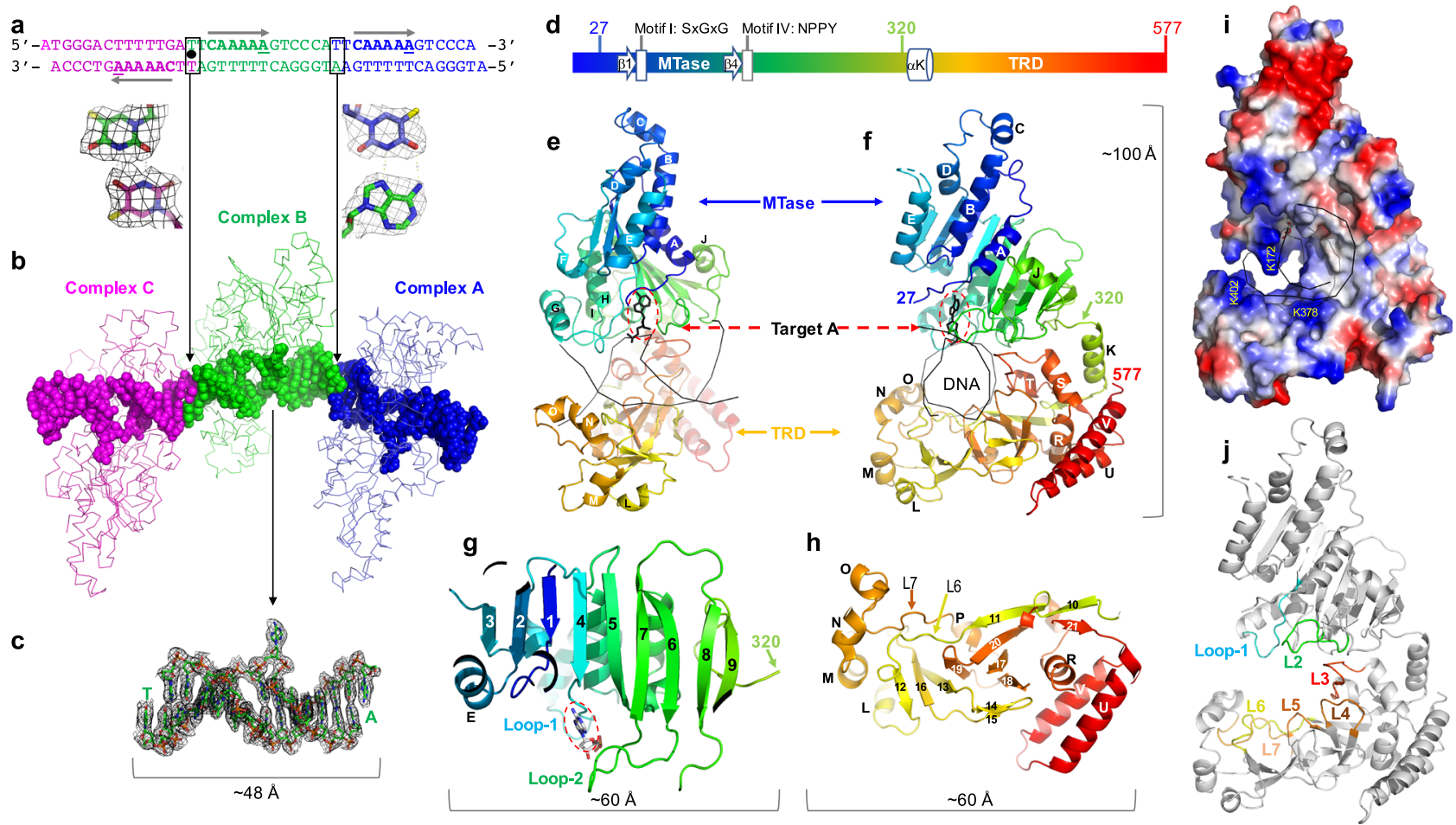

Fig. 2 Structure of CamA-DNA complex. a DNA oligos used for co-crystallization are connected by two different joints in the crystals (T:T mismatch linking complexes $B$ and $C$, and $T: A$ in a Hoogsteen base pair linking complexes $A$ and $B$ ). The CamA recognition sites (CAAAAA) are indicated by arrows from $5^{\prime}$ to $3^{\prime}$, and the three target adenines are each separated by 1.5 turns of DNA (14 bp). b Three CamA-DNA complexes were formed in the crystallographic asymmetric unit. c Omit electron density map (contoured at $4.5 \sigma$ above the mean) for the DNA molecule of complex B. The base-flipped adenine is visible. $\mathbf{d}$ Schematic illustration of CamA with relative locations of motifs I and IV and TRD. Residue 320 before $\alpha \mathrm{K}$ indicates the linker point between the two domains. e, $\mathbf{f}$ Two orthogonal views of CamA, colored in spectrum from blue ( $\mathrm{N}$-terminus) to red (C-terminus). $\mathbf{g}$ The $\mathrm{N}$-terminal catalytic domain folds into a nine-stranded sheet. Loop- 1 follows strand $\beta 4$ and loop- 2 is between strands $\beta 6$ and $\beta 7$. h Antiparallel $\beta$-structure of C-terminal TRD. i Electrostatic surface of CamA with blue for positive and red for negative charges. $\mathbf{j}$ Seven loops (L1-L7) line the basic surface of the cleft for DNA binding.

The $K_{\mathrm{D}}$ values for SAM and sinefungin are in approximately the same range as the $K_{\mathrm{m}}$ value for SAM $(>17 \mu \mathrm{M})$. For comparison, M.TaqI has the binding order preference of sinefungin $\left(K_{\mathrm{D}}=\right.$ $0.34 \mu \mathrm{M})>$ SAM $(2.0 \mu \mathrm{M})>$ SAH $(2.4 \mu \mathrm{M})^{32}$. While the actual $K_{\mathrm{D}}$ values were determined under different conditions, the relative binding preferences can be compared, and most strikingly show CamA (unlike M.TaqI) to prefer SAH binding to that of SAM.

Next, we measured the binding affinity of CamA to substrate DNA in the absence and presence of sinefungin or SAH. First, CamA exhibited DNA binding affinity dependent on ionic strength, with $K_{\mathrm{D}}$ value of $40 \mathrm{nM}$ at $150 \mathrm{mM} \mathrm{NaCl}$ increasing to $\sim 0.2 \mu \mathrm{M}$ at $250 \mathrm{mM} \mathrm{NaCl}$ (indicating decreased binding; Supplementary Fig. 3a). Second, compared to absence of cofactor, CamA demonstrated about $1.8 \times$ stronger binding to substrate DNA in the presence of sinefungin (from 0.20 to $0.11 \mu \mathrm{M}$ ), whereas the presence of SAH yielded about $1.8 \times$ weaker binding to DNA (from 0.20 to $0.37 \mu \mathrm{M}$; Fig. 1e). The opposite effects of sinefungin and SAH on DNA binding are small but repeatable (Supplementary Fig. 3b, c), and might represent two CamA reaction states (substrate complex before, and product complex after, methyltransfer). Like SAM, sinefungin also carries a formal positive charge, but does not support methyl transfer, and may thus mimic the pre-transfer state.

Structure of CamA in complex with substrate DNA. We next sought to understand how CamA recognizes the substrate DNA sequence, and how it specifically methylates the adenine five nucleotides away from the $5^{\prime} \mathrm{C}: \mathrm{G}$ base pair in CAAAAA. Accordingly, we purified CamA-DNA complexes (Supplementary
Fig. 4a, b) and grew co-crystals, using a 13-base pair duplex containing a centrally-located A:T and with a $5^{\prime}$-overhang at each end (either an A or T) (Fig. 2a). The complex crystallized in space group $P 2_{1} 2_{1} 2_{1}$, resulting in a structure determined to a resolution of $2.68 \AA$ (Supplementary Table 1). Although CamA protein contains all nine amino acid motifs conserved in the family of DNA adenine MTases ${ }^{24,33}$, molecular replacement was not successful using homolog models. We therefore used experimental phasing, from anomalous signals of selenomethionyl-substituted CamA, for de novo structure determination ${ }^{34}$ (Supplementary Table 1 and Supplementary Fig. 4c).

In the crystallographic asymmetric unit, there are three CamADNA complexes (A, B and C) (Fig. 2b). The DNA molecules of complexes $A$ and $B$ are stacked head-to-tail with the $5^{\prime}$ overhanging bases forming an A:T base pair in the joint (Fig. 2b). In contrast, the DNA molecules of complexes $\mathrm{B}$ and $\mathrm{C}$ are rotated $180^{\circ}$ in regard to each other, forming a T:T mismatch in the joint of DNA molecules (Fig. 2b). Thus, the three DNA segments form a pseudo-continuous dsDNA molecule, with two target $\mathbf{A}$ residues located on the same strand, while the third target $\underline{\mathbf{A}} \overline{\text { is }}$ located on the opposite strand, in a way representing the presence of methylated CAAAAA on both sense and antisense strands in the genomes of $C$. $\overline{\text { difficile isolates }}{ }^{24}$. All DNA base pairs, including the joints were observed, in the electron density (Fig. 2c).

The protein components of the three complexes do not directly contact one another, and are highly similar in conformation (Supplementary Fig. 5). Pairwise comparisons revealed rootmean-square deviations of just $0.3-0.5 \AA ̊$ between $\sim 550$ pairs of $\mathrm{Ca}$ atoms, and so we describe only complex B hereafter. CamA 
comprises an N-terminal catalytic domain (residues 1-320) and C-terminal target recognition domain (TRD; residues 320-577) (Fig. $2 \mathrm{~d}-\mathrm{f}$ ). The TRED is the region of DNA MTases responsible for DNA sequence specificity 35,36 . The first 26 residues are disordered in the current structure. The catalytic domain is consistent with structures of Class I MTases ${ }^{37}$, though most such MTases include seven $\beta$-strands, CamA contains a nine stranded $\beta$-sheet ( $\beta 1-\beta 9$ in Fig. $2 \mathrm{~g}$, with $\beta 8-\beta 9$ being the added ones). The structure includes six helices ( $\alpha \mathrm{A}-\alpha \mathrm{E}$ and $\alpha \mathrm{J})$ located on the front side of the sheet and four helices $(\alpha \mathrm{F}-\alpha \mathrm{I})$ on the back side of the sheet, resulting in an open $\alpha \beta a$ sandwich ${ }^{37-39}$ (Fig. 2e, f). As is also typical of Class I structures, there is a central topological switch point between strands $\beta 1$ and $\beta 4$, where SAM and the target Ade generally binds at the carboxyl ends of these two parallel neighboring strands. In addition to a characteristic reversed $\beta$ hairpin ( $\beta 6$ and $\beta 7$ ) next to strand $\beta 5$, CamA contains a second consecutive $\beta$ hairpin ( $\beta 8$ and $\beta 9$ ) at the same end of the sheet (Fig. 2g). In this respect, the structure resembles that of the adenine MTase of the TaqI restriction-modification system (PDB $2 \mathrm{ADM})^{40-42}$. The extra strands make the N-terminal catalytic domain larger, with the longest dimension $(\sim 60 \AA)$ comparable to that of the larger TRD domain (Fig. $2 \mathrm{~g}, \mathrm{~h}$ ), and are stabilized via interdomain interactions.

The N-terminal catalytic domain is connected to the Cterminal TRD domain via a linker centered at residue 320 (Fig. $2 \mathrm{f}$ and Supplementary Fig. 6a). The TRD domain is folded into 12 strands $(\beta 10-\beta 21)$, forming a series of antiparallel strands in a $\beta$-cluster (Fig. 2 h). Four short helices $(\alpha \mathrm{L}-\alpha \mathrm{O})$ flank this $\beta$-cluster structure on one side, while on the other side of the $\beta$-structure are six more helices ( $\alpha \mathrm{Q}-\alpha \mathrm{V})$ (Fig. 2h). The basic surface of the cleft between the two domains forms the DNA binding site with residues from both domains approaching DNA from opposite directions (Fig. 2i).

Distortion of DNA conformation. The CamA-bound DNA molecule undergoes major distortions at the 6-bp recognition site (labeled as 1-6), while the unrecognized flanking sequence maintains B-form (Fig. 3a). We generated a regular B-DNA model of the same sequence, and superimposed it onto the CamA-bound DNA (Fig. 3a). Several substantial distortions were observed. First, the bound DNA molecule is kinked between base pairs 6 and 7 (the joint between target $\underline{\mathbf{A}} 6$ and the $3^{\prime}$ G7) and bent $\sim 30^{\circ}$ (Fig. 3b), resulting in the largest movement of $\sim 23 \AA$ towards one end of the DNA molecule (Fig. 3c). Second, the six recognized base pairs have conformational features of base-pair propeller twist (C1:G1 and A3:T3), base-pair buckle (A4:T4), and base pair-rearrangement (A5:T6) (Fig. 3d). The space left by the rearranged A5 is partially occupied by protein residue Lys172, which forms a hydrogen bond (H-bond) with the orphaned base T5. Third, the $\pi$-stacking force in the non-target strand is disrupted by Tyr455, which is wedged between T5-T6 (Fig. 3e). Fourth, the target A6 is flipped completely out of the helix (Fig. 3f).

While the extrahelical positioning of the target A6 base is expected, based on numerous studies with other DNA MTases $^{42-45}$, the squeezing between the rearranged A5 and G7the two nucleotides immediately flanking the target A6-is to date unique to CamA. The three phosphate groups, one $5^{\prime}\left(\mathrm{P}_{-1}\right)$ and
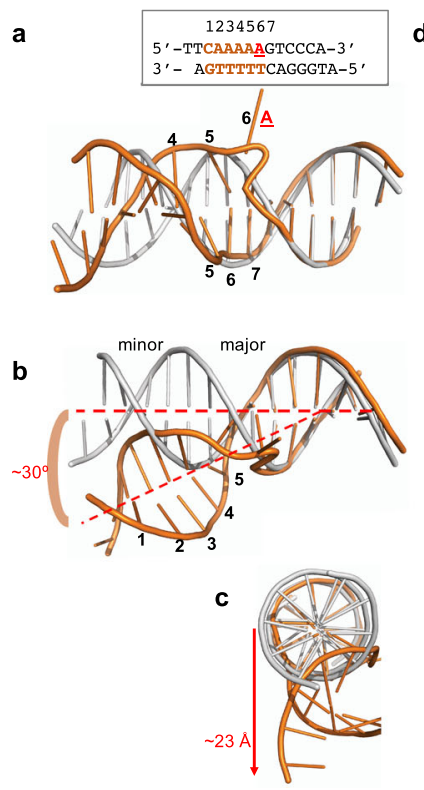
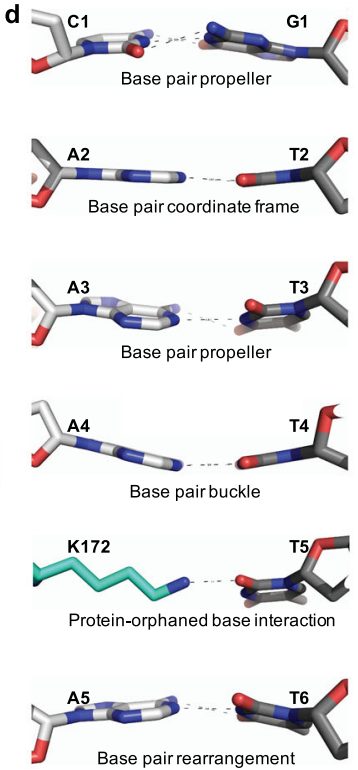
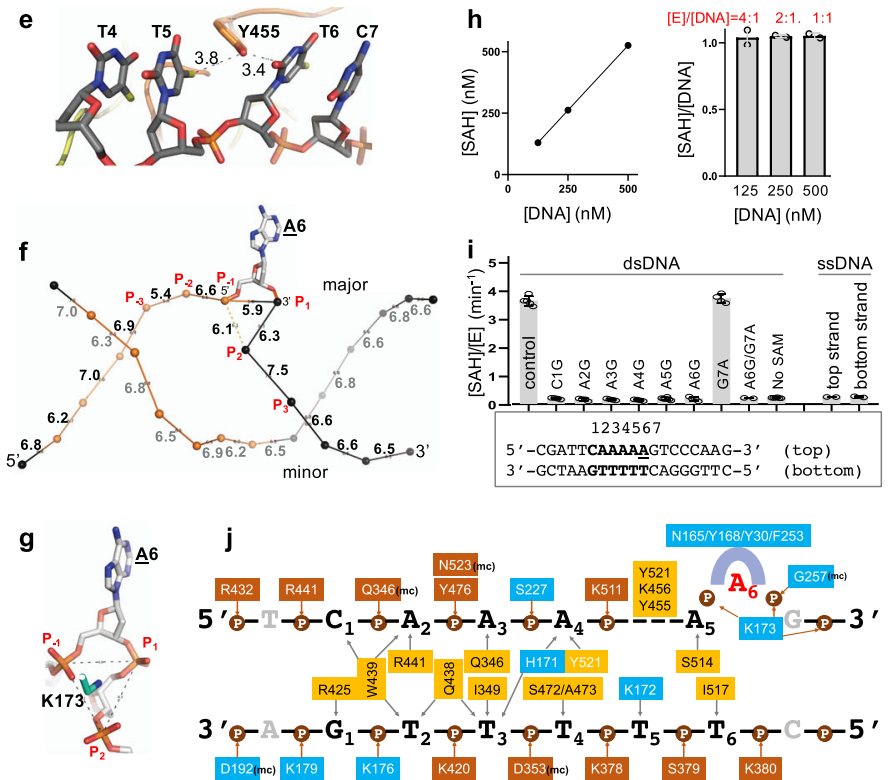

Fig. 3 CamA-bound DNA conformation. a The 6-bp recognition sequence is numbered from 1 to 6 . B-form DNA (gray) is superimposed with CamA-bound DNA molecule (orange). Note the flipped-out A6 and rearranged base pairs between A5 and T6. b-c Conformational deviations of CamA-bound DNA molecule from B-form DNA. d Conformational differences among the six base pairs of the recognition sequence. e Discontinuous $\pi$-stacking between T5 and T6. $\mathbf{f}$ Inter-phosphate distances of CamA-bound DNA with the largest variations from three phosphate groups $5^{\prime}$ and $3^{\prime}$ respectively to the target adenine $\left(P_{-3}\right.$ and $\left.P_{3}\right) \cdot \mathbf{g}$ Lys173 sits in the center of equilateral triangle of phosphate groups $P_{-1}, P_{1}$, and $P_{2}$. $\mathbf{h}$ Under the single turnover conditions where the enzyme is present at or above the DNA substrate concentration, only one methylation event occurs ( $N=2$ ). Enzyme concentrations used are within a linear range (left). Source data are provided as a Source Data file. i Effects of single-base pair substitutions. No activity was observed for ssDNA, using the unsubstituted and unannealed strands separately. Data represent the mean \pm SD of $N$ number of independent determinations $(N=5$ for control, $N=4$ each for C1G, A2G, A3G, A4G, A6G, and G7A substitutions, N=6 each for A5G and No SAM control, and N=2 each for A6G/G7A and ssDNA substrates) performed in duplicate. Source data are provided as a Source Data file. $\mathbf{j}$ Schematic illustration of CamA-DNA interactions (for an enlarged version, see Supplementary Fig. 6b): residues in cyan background are from the N-terminal catalytic domain, and residues in (light and dark) orange from the C-terminal TRD. The base-specific contacts are placed between the two strands and the phosphate contacts are depicted above or below the strand. mc, main-chain-atom-mediated contacts. 
a
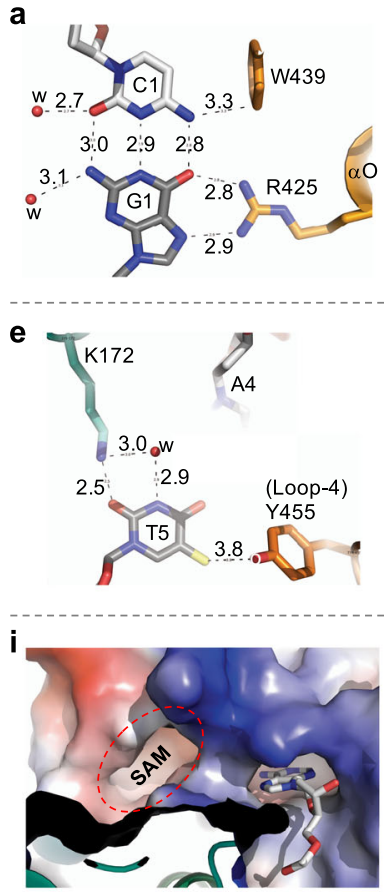

b

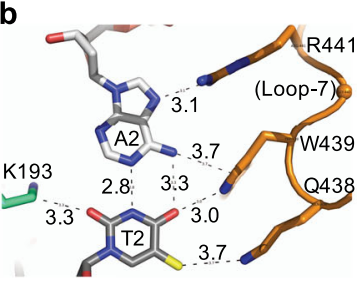

f

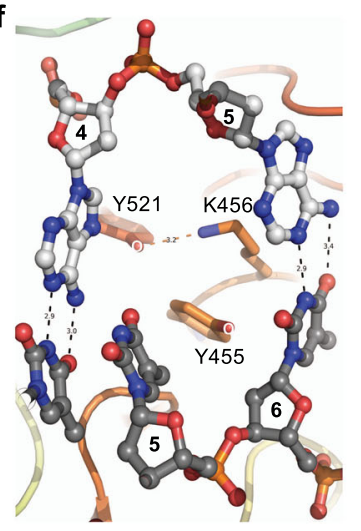

c

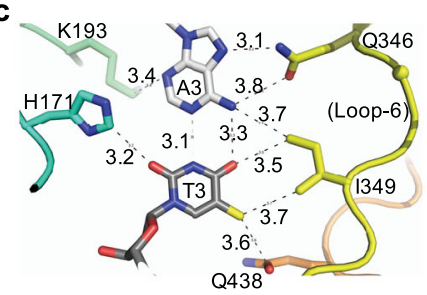

g

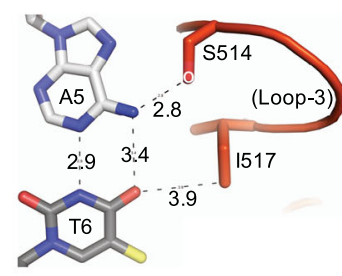

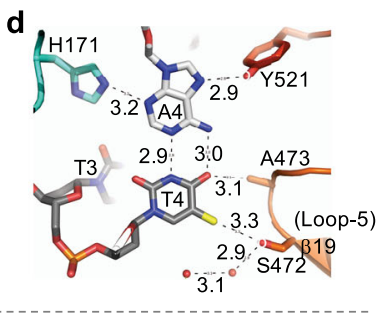

h

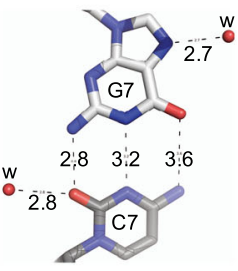

j

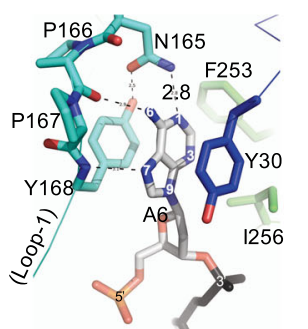

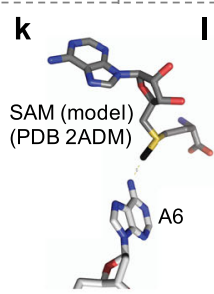

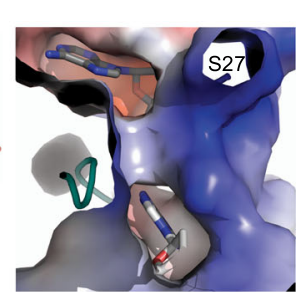

Fig. 4 CamA-mediated base-specific recognition. a Interactions with C1:G1 base pair. Interatomic distances are shown in angstroms. b-d Interactions with A2:T2, A3:T3, and A4:T4 base pairs at both major (right) and minor (left) grooves. e Interactions with the orphan T5. f View from the DNA minor groove, with intercalation of TRD residues that occupy the space normally occupied by A5. $\mathbf{g}$ Interactions with the rearranged A5:T6 base pair. $\mathbf{h}$ Lack of proteinmediated contacts with G7:C7 base pair. $\mathbf{i}$ The flipped-out A6 bound in the active-site cage. The nearby SAM-binding pocket is empty in the current structure. $\mathbf{j}$ Interaction with the target A6 in the aromatic cage. $\mathbf{k}$, I A modeled SAM molecule in the cofactor binding pocket. Label S27 indicates the first ordered residue in the current structure.

two $3^{\prime}\left(\mathrm{P}_{1}\right.$ and $\left.\mathrm{P}_{2}\right)$ to the target $\mathrm{A}$, yield an equilateral triangular conformation with inter-phosphate distances of $\sim 6 \AA$ (actually 5.9 , 6.1, and $6.3 \AA$ A Fig. 3f). These three phosphate groups are balanced by the positively charged Lys173 sitting in the center of the triangle (Fig. 3g), adjacent to the gap-filling Lys172. Currently, we do not know how these events unfold temporally, leading to the observed distortions.

It is interesting that the CamA recognition sequence contains five adenines (CAAAAA). We first confirmed that, with a DNA substrate having a single occurrence of CAAAAA, only one methylation event occurs under the single turnover conditions with CamA concentration at or higher than that of the DNA substrate (Fig. 3h). We mutated each base pair of the recognition sequence to G:C individually and observed no methyl transfer activity, whereas the substitution of a base pair immediately outside of the recognition sequence had no effect on CamA activity (Fig. 3i).

Base specific interactions. Seven loops, two from the catalytic domain and five from the TRD domain, face the DNA, and provide most of the functionally important residues recognizing the six base pairs on both strands (Fig. $2 \mathrm{j}$ ). There are extensive protein-phosphate interactions, involving many basic residues across both strands of DNA, that form an interface with the 6-bp recognition sequence (Fig. $3 \mathrm{j}$ and Supplementary Fig. 6b), and that likely stabilize the distorted protein-DNA complex. This involvement of the catalytic domain in sequence recognition is not that unusual among DNA MTases, as illustrated (for example) by M.SinI via mutagenesis ${ }^{46}$ and CcrM by structure ${ }^{45}$.

The guanine of the first C1:G1 base pair is recognized by CamA Arg425, which forms bidentate H-bonds with the $\mathrm{O} 6$ and
N7 atoms of the G1 base (Fig. 4a), in accordance with the most common mechanism for guanine recognition ${ }^{47,48}$. In addition, the $\mathrm{N} 4$ atom of $\mathrm{C} 1$ base is in van der Waals contact with Trp439, which in turn contacts the next base pair A2:T2 (Fig. 4b). Adenine A2 forms a H-bond with Arg441 (Fig. 4b), while Gln438 bridges between the two methyl groups of thymine residues T2 and T3 (Fig. 4b, c).

Like G1, adenine A3 is recognized by $\mathrm{Gln} 346$ via bidentate $\mathrm{H}$ bonds (Fig. 4c). Juxtaposition of Gln (or Asn) with adenine is a common mechanism for adenine recognition ${ }^{47}$, as occurs, for example, with Gln418 of CTCF ${ }^{49}$, or Gln264, Asn285 and Gln350 of ZNF410 $0^{50}$. In such cases, the side chain carboxamide moiety of glutamine or asparagine generally donates one $\mathrm{H}$-bond to adenine N7 and accepts one from adenine N6, respectively, a pattern specific to Ade. However, the side chain of Gln346 is rotated out of the plane of adenine base, with the interatomic distance $(3.8 \AA)$ longer between the side-chain oxygen atom and the adenine N6 atom (Fig. 4c). The weakened H-bond interaction is probably due to the neighboring residue Ile349, which sits in the major groove edge of A3:T3 and forms van der Waals contacts with the base pair (Fig. 4c). On the minor groove side, His171 provides a $\mathrm{C}-\mathrm{H} . . . \mathrm{O}$ type bond ${ }^{51}$ with $\mathrm{O} 2$ of $\mathrm{T} 3$ base (Fig. 4c). We note that the H-bonding capacity of base pair A3:T3 is fully saturated in the major and minor grooves.

Like A3:T3, the next base pair A4:T4 is also engaged in interactions in both the major and minor grooves (Fig. 4d). Adenine A4 is bordered by His171 (minor groove) and Tyr521 (major groove). Thymine T4 is engaged in two $\mathrm{C}-\mathrm{H} \cdots 0$ type bonds with Ser472 (interacting with the C5 methyl group) and Ala473 (interacting with the O4 oxygen). Thus, the first four base pairs (C1:G1 to A4:T4) are engaged in extensive direct protein-DNA interactions in both grooves. In addition to single 
base-specific interactions, such as Arg425-G1 and Asn346-A3, three residues each bridge between two base pairs, Trp439 between $\mathrm{C} 1$ and $\mathrm{A} 2, \mathrm{Gln} 438$ between T2 and T3 and His171 between $\mathrm{T} 3$ and A4. In addition, one aromatic (Trp439) and three hydrophobic residues, Ile349, Ala473, and Ile517 (see below), are located intimately at the protein-DNA interface. A tryptophan or histidine spanning two base pairs have been observed previously in ZNF410 and ZFP568, respectively ${ }^{50,52}$.

As noted above, the orphaned $\mathrm{T} 5$ forms a $\mathrm{H}$-bond via its $\mathrm{O} 2$ atom with Lys172 approaching from the minor groove (Fig. 4e). In addition, Tyr455 approaches from the major groove and makes a weak van der Waals contact with the T5 methyl group. Looking through the DNA, from the minor groove side to the major groove side, two additional protein residues, Lys456 and Tyr521, wedge their side chains into the DNA and push adenine A5 sideways, to the base pairing position initially occupied by target A6, which effectively has been squeezed out (Fig. 4f). The rearranged A5:T6 base pair has two contacts with protein side chains of Ser514 and Ile517 (Fig. 4g); the number of contacts seems to be fewer than what was observed for the first base pairs. As shown by diminished enzymatic activity on substrates having base pair substitution of A5:T5 to G5:C5, a guanine at position 5 would generate a G:T mismatch for the rearranged pair. Beyond the recognition sequence, there is no protein-mediated DNA base contact (Fig. 4h). In sum, the discrimination yielding sequence specificity for CAAAA A is provided by base-specific interactions with the first four base pairs of both strands, involving hydrogen bonds and van der Waals contacts in both grooves, and by the base pairing of the rearranged A5:T6. By examining $C$. difficile CamA orthologs that were complete but nonidentical to that from strain 630 (on which this study is based), we note that every identified residue involved in DNA base interactions is completely conserved (Supplementary Fig. 7); so we would expect the sequence specificity to be unchanged as well.

"Squeezed out" target adenine. Like other structurally characterized DNA MTases ${ }^{42-45}$, the target adenine A6 is flipped out and inserted into an active-site cage (Fig. 4i) formed by three aromatic residues (Fig. 4j). The adenine is stacked in-between Tyr30 and Tyr168, and sealed off with Phe253 in the bottom of the cage (Fig. 4j). Tyr168 is a part of the NPPY motif, a catalytically active-site sequence (motif IV) conserved among amino MTases $^{33}$. The polar groups of the target adenine ring (N1, N6, and N7), that normally form the Watson-Crick pair with thymine and/or interact with protein in the major groove, are now involved in hydrogen bonds with the side chain amide group of Asn165 (interacting with N1 atom), main-chain carbonyl oxygen of Pro166 (interacting with N6 amino group), and main-chain amide nitrogen of Tyr168 (interacting with N7 atom) (Fig. 4j). This pattern of hydrogen bonding defines the specificity for adenine in the active-site binding cage.

Cofactor-induced conformational change. Intriguingly, in the CamA-DNA cocrystal structure, we did not observe a bound cofactor in the SAM binding pocket next to the active-site (Fig. 4i), even though exogenous SAH was added during initial complex formation. However, the added SAH had dissociated during subsequent complex purification (Supplementary Fig. 4a, b), probably due to the low binding affinity. Nevertheless, we modeled in a SAM and positioned the methyl group and sulfur atom of SAM in line with the target N6 atom (Fig. 4k). This linear arrangement, comprising the nucleophile, the methyl group and the leaving thioester group in the transition state, is required for the $S_{N} 2$ reaction mechanism used by SAM-dependent MTases ${ }^{37}$. The model exhibits a good fit, with no apparent clashes (Fig. 4l).
However, nothing in the current model obviously explained the relatively low SAM affinity of CamA.

The absence of cofactor might also explain the disorder of the $\mathrm{N}$-terminal residues (amino acids 1-26), which is near the binding pocket (the first ordered residue S27 is labeled in Fig. 4l). Knowing CamA has weak binding affinity for its cofactor, we repeated the crystallization, this time including $\mathrm{SAH}$ throughout the preparation of CamA-DNA-SAH ternary complex. We started with a molar ratio of 1:24 of CamA:SAH, and ended with a final 1:3 ratio of concentrated complex for crystallization (see "Methods" section). The ternary complex was crystallized under conditions similar to those used for the binary CamADNA complex, and the structure was determined to a slightly higher resolution of $2.54 \AA$ (Supplementary Table 1). We observed bound SAH in the carboxyl ends of strands $\beta 1-\beta 3$ (as expected), together with ordered residues of nearly the entire $\mathrm{N}$ terminal fragment starting from residue 2 (Fig. 5a, b). The ordered N-terminal fragment forms two additional helices (residues 9-18 and residues 21-27) and closes off the cofactor binding pocket (Fig. $5 \mathrm{c}-\mathrm{e}$ ). Under some circumstances, this coupled conformational rearrangement might contribute to the low binding affinity of cofactor (SAM, SAH, or sinefungin), though this remains to be tested.

CamA makes extensive contacts with all three moieties of SAH: the adenine ring, the ribose, and the homocysteine. Among the residues interacting with $\mathrm{SAH}$ (see below), there are three aspartates, Asp60 of motif I (the last residue of strand $\beta 1$ ), Asp114 of motif II (the last residue of strand $\beta 2$ ) and Asp149 of motif III (the first residue of helix $\alpha \mathrm{F}$ connected to the carboxyl end of strand $\beta 3$ ) (Supplementary Fig. 6a). The three negatively charged residues are responsible for binding three moieties of $\mathrm{SAH}$, respectively, the terminal amino group, the ribose hydroxyl oxygen atoms, and the exocyclic amino group of adenine ring.

The adenosyl moiety of SAH forms H-bonds with Asp149 (via its N6 atom) and the main chain amide nitrogen of Ser150 (via its $\mathrm{N} 1$ atom) (Fig. 5f). In addition, an ethylene glycol molecule (used as the cryoprotectant during crystal freezing) forms two $\mathrm{H}$-bonds with the N6 and N7 atoms, respectively. Furthermore, the SAH adenine ring is stacked between Ile115 and Phe200 (Fig. 5g).

The ribose moiety of SAH engages in five types of interactions. Its hydroxyl groups $\left(2^{\prime}-\mathrm{OH}\right.$ and $\left.3^{\prime}-\mathrm{OH}\right)$ interact with Asp114 (Fig. 5f). The 5-membered ribose ring stacks with the 5membered pyrrolidine ring of Prol67 (Fig. 5g). The $3^{\prime}-\mathrm{OH}$ group has a water-mediated interaction with Tyr31 (Fig. 5h). The carbon $\mathrm{C}^{\prime}$ is in van der Waals contact with the mainchain carbonyl oxygen atom of Ser27 (Fig. 5h). Fifth among the ribose interactions, the ring $\mathrm{O}^{\prime}$ oxygen forms a $\mathrm{C}-\mathrm{H} \bullet \bullet \mathrm{O}$ type interaction with the side chain carbon $C \beta$ atom of Ser62 (Fig. 5i). Ser62 is part of motif $\mathrm{I}$, in the position normally occupied by Phe or Tyr, as discussed below.

Finally, we describe interactions with the aminocarboxypropyl moiety of SAH. This moiety forms an electrostatic interaction with Asp60, H-bonds with Thr32, and van der Waals contact with Tyr31 (Fig. 5j). The sulfur atom of SAH, where a transferable methyl group would be attached in SAM, is in an appropriate distance $(3.8 \AA)$ and orientation from the target N6 atom of Ade of DNA - the methyl acceptor (Fig. 5k). The distance between the sulfur of SAH and the N6 of target Ade is approximately the sum of the bond distance of donor-methyl $\left(\mathrm{S}^{+}-\mathrm{CH}_{3}=1.82 \AA\right)$ and the bond distance of acceptor-methyl $\left(\mathrm{CH}_{3}-\mathrm{N}=1.5 \AA\right)$. The H-bonding interactions with the mainchain carbonyl oxygen atoms of Gly 28 and Pro166 could facilitate the deprotonation of the target amino group of Ade during catalysis.

Besides binding $\mathrm{SAH}$, the ordered $\mathrm{N}$-terminal residues provided minimal contacts with DNA, with one noticeable exception. Lys25 

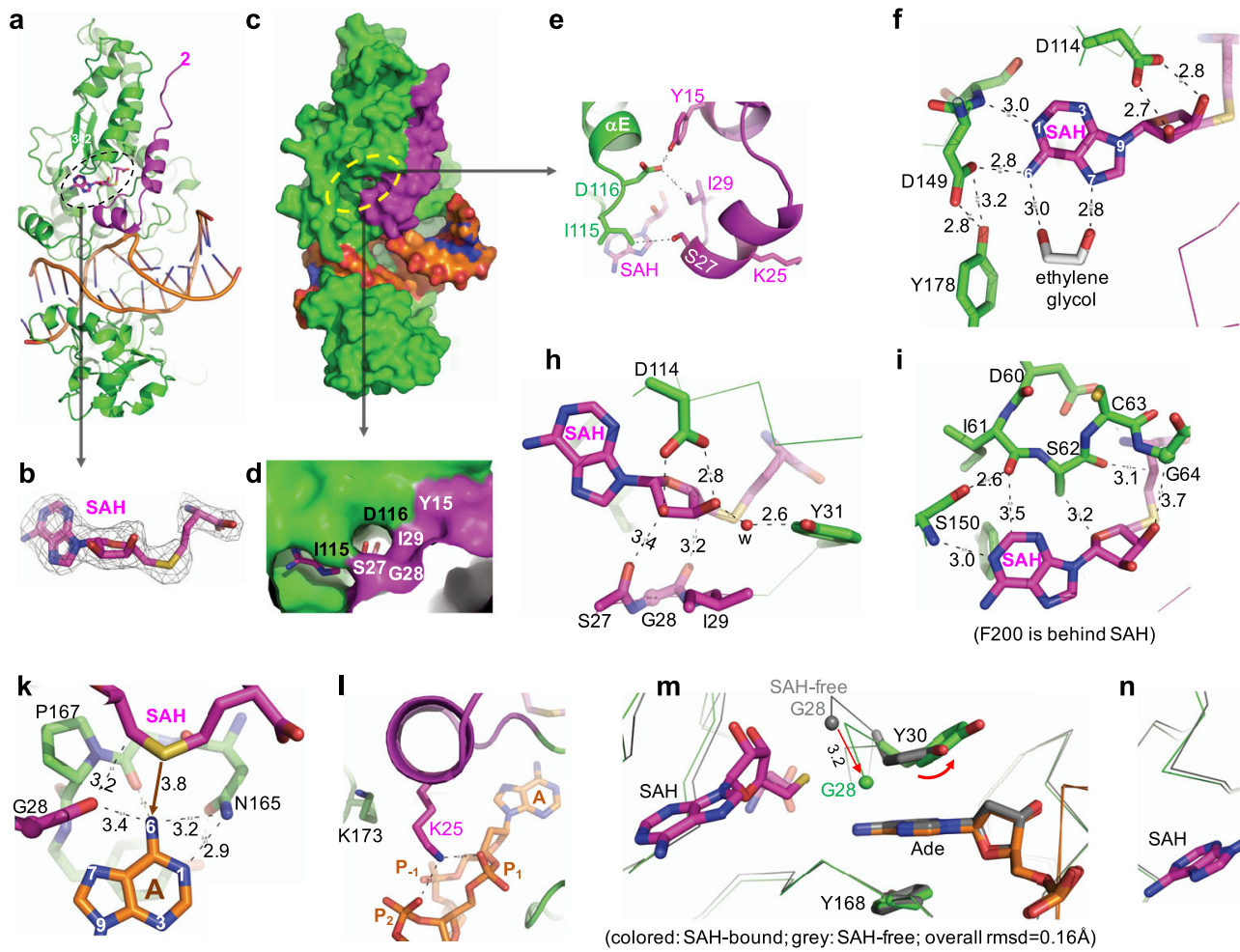

g
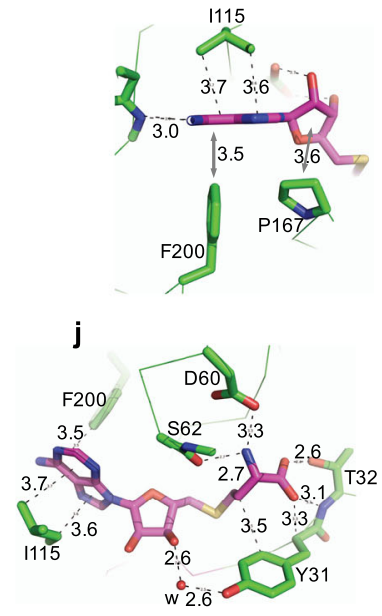

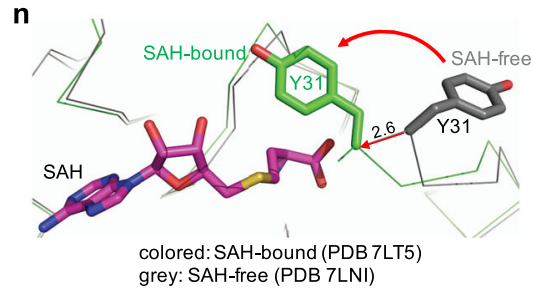

Fig. 5 Cofactor-induced conformational change. a The ternary structure of CamA-DNA-SAH (in stick model). The ordered N-terminal residues are in magenta. b The omit electron density map (contoured at 5.0 $\sigma$ above the mean) for the bound SAH. c Surface presentation of the ternary complex of SAH (in green and magenta), DNA (in orange) and SAH. d The enlarged binding pocket of SAH, where the edge of adenine moiety and the ribose hydroxyl groups were visible. $\mathbf{e}$ Intramolecular interactions between the $\mathrm{N}$-terminal residues (in magenta) and the loop prior to helix $\alpha \mathrm{E}$. $\mathbf{f}, \mathbf{g}$ Interactions with $\mathrm{SAH}$ adenine moiety. $\mathbf{h}$ Interactions with SAH ribose moiety. $\mathbf{i}$ Interactions involving residues of motif I. Note that Phe200 is behind SAH and away from the viewer. $\mathbf{j}$ Interaction with SAH aminocarboxypropyl moiety. $\mathbf{k}$ Interactions between the methyl donor (sulfur atom of SAH) and methyl acceptor ( $\mathrm{N6}$ of target Ade). I Conformational change of Lys25 and Lys173. $\mathbf{m}$ Superimposition of active sites of complexes B, in the SAH-free (PDB 7LNI) and SAH-bound (PDB 7LT5) states. The bound target adenine is sandwiched between two tyrosine residues, with Tyr30 undergoing a small rotation. $\mathbf{n}$ The largest movement is Gly28 and Tyr31, which move from an open to the closed conformation upon SAH binding.

interacts with the triangular conformation of three phosphate groups surrounding the target A6 (Fig. 5l). The Lys25-phosphate interaction effectively replaced Lys173, which would be placed in the center of the triangle in the absence of the ordered N-terminal residues (see Fig. 3g). The interplay between the two lysine residues, Lys 25 and Lys173, allows CamA to bind DNA even in the absence of bound cofactor, but does not gain additional protein-DNA interactions. While the DNA interface is largely the same in the SAH-free and SAH-bound states, the N-terminal residue Tyr30 involved in binding of the flipped target adenine undergoes a small rotation from the nearly perfect stacking between the two rings of Tyr30 and the target Ade in the SAH-free state to a dislodged angle (Fig. $5 \mathrm{~m}$ ). Accompanying this small movement involved in DNA interaction is the large movement of Gly28 and Tyr31, which close off the SAH binding pocket (Fig. 5n).

Comparison with other orphan methyltransferases. Within Class I MTases, CamA belongs to the $\gamma$-group ${ }^{33}$, based on the sequential order of conserved sequence motifs, particularly sequences for binding the methyl donor SAM (motif I after strand $\beta 1$ ) and for catalysis (motif IV after strand $\beta 4$ ), and the location of the TRD domain in relation to these two motifs (Fig. 6a, b). As noted, CamA is an orphan MTase, meaning it is not paired with a restriction endonuclease. Examples of such orphan MTases include the DNA adenine MTase (Dam) in Escherichia coli (Gammaproteobacteria) and cell cycle-regulated DNA MTase (CcrM) in Caulobacter crescentus (Alphaproteobacteria) which are, respectively, responsible for post-replication maintenance of daughter strand adenine methylation at two very similar sequences: GATC or GAnTC $(\mathrm{n}=\text { any nucleotide })^{21,53}$. Based on the different order of motifs, and the location of the TRD, Dam is a member of a-group and CcrM is a $\beta$-group MTase. Taken together, our kinetic and structural characterization of Dam, CcrM, and CamA means that we have characterized an orphan MTase from each group $(\alpha, \beta \text {, and } \gamma)^{45,54-56}$

Examining the three orphan MTases altogether, there are similarities and major differences among the three enzymes. First, Dam ( $\alpha$ group) and CamA ( $\gamma$ group) are active as monomers, having DNA recognition and methylation functions in a single polypeptide, while CcrM ( $\beta$ group) also has both functions in one polypeptide but is only active as a homodimer. The requirement for a dimeric form is unique to the group $\beta$ MTases due to the relative positions of the functional domains, allowing the enzyme to use division of labor between two subunits in terms of DNA binding and methylation ${ }^{44,57}$.

Second, except for the flipped-out target adenine, the Dambound DNA conformation has intact intrahelical paired bases. In contrast, CcrM pulls the two DNA strands apart, creating a bubble comprising four enzyme-recognized, unpaired bases, and CamA squeezes out the target adenine by base pair rearrangement (Fig. 6c-e).

Third, all three enzymes use an arginine to interact with a $5^{\prime}$ guanine (Fig. $6 \mathrm{f}-\mathrm{h}$ ) and the Arg-Gua interaction makes sequencediscriminatory contacts in both Dam and CcrM, and most likely also for CamA as shown by the diminished activity of a G1 to 
a

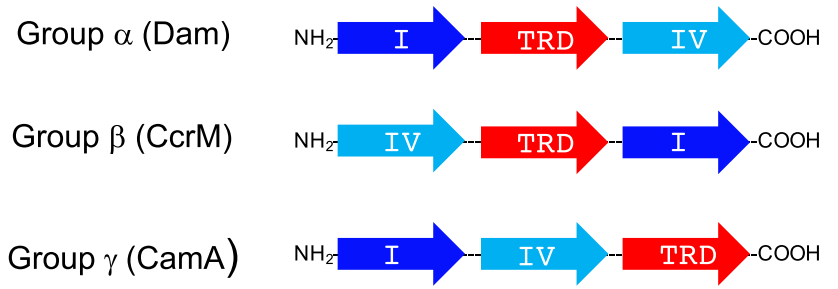

b

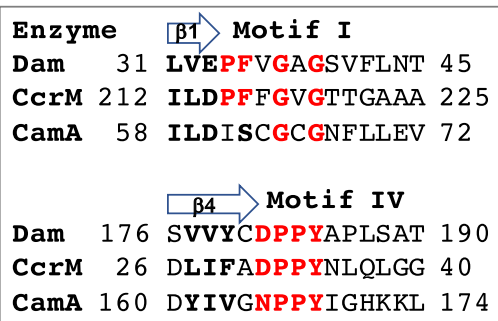

C

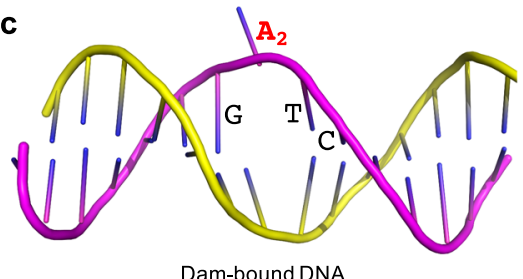

f

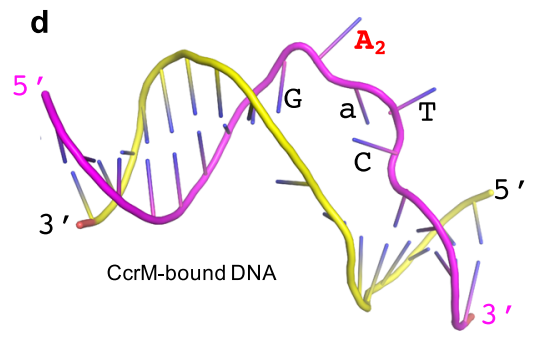

$\mathbf{g}$

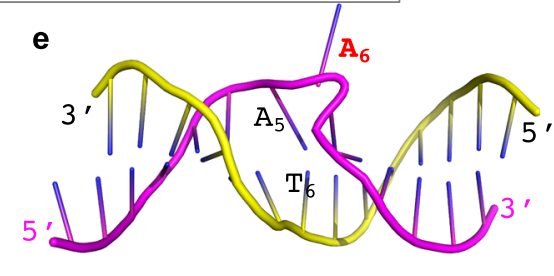

CamA-bound DNA h

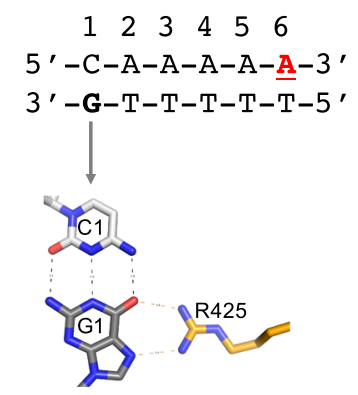

Fig. 6 Comparison of three orphan adenine methyltransferases. a Schematic of three Class I groups of amino-MTases, showing altered orders of motifs responsible for SAM binding (motif I), catalysis (motif IV) and target DNA substrate binding (TRD). b Sequence alignment of motif I and motif IV for the three orphan adenine MTases. c-e Enzyme-bound DNA conformations in Dam (c), CcrM (d), and CamA (e). f Dam interacts with guanine G4 of the nontarget strand. $\mathbf{g}$ CcrM interacts with guanine $\mathrm{G} 1$ of the target strand. The lowercase $\mathrm{m}$ in black circle is the methylated adenine of the parental strand immediately after DNA replication. $\mathbf{h}$ CamA interacts with guanine $\mathrm{G} 1$ of the non-target strand. The underlined $\underline{\mathrm{A}}$ in red in each case is the methylation target.

C1 substitution (see Fig. 3i). However, these contacts are made to different strands relative to the substrate Ade. In Dam and CamA, the recognized Gua is on the opposite strand from the target Ade; whereas in CcrM, the recognized Gua is on the same strand as, and adjacent to, the target Ade.

Fourth, Dam and CamA make base-specific contacts to both DNA strands, whereas CcrM contacts bases only in the target strand $^{45}$. These two features of CcrM, strand separation and base recognition on the same strand that contains the target Ade, allow CcrM (but not Dam) to methylate both double-stranded (ds) and single-stranded (ss) $\mathrm{DNA}^{27}$. Like Dam, CamA is inactive on ssDNA (Fig. 3i).

Fifth, the substrates of Dam and CcrM are hemimethylated symmetrical (GATC) or gapped-symmetrical (GAnTC) sequences of newly replicated DNA, and most (if not all) target adenines are located-following replication-on the same (daughter) strand in a uniform direction, allowing the enzymes to be highly processive $26,27,58$. In contrast, CamA methylates an asymmetric 6-bp sequence, and methylation sequence targets in a given chromosome region can occur on either strand ${ }^{24}$. To achieve this, CamA would either slide along the DNA to recognize and methylate the target adenines on the same strand (as illustrated by complexes A and B in Fig. 2a, b); or release from the DNA, turn around, and rebind to methylate the opposite strand (as suggested by comparing complexes $\mathrm{B}$ and $\mathrm{C}$ in Fig. 2a, b).

Finally, there is one notable difference in the sequence of motif I for binding SAM. In both Dam and CcrM, motif I contains FxGxG (Fig. 6b) - the widespread motif ${ }^{59}$ conserved among many SAM-dependent MTases generating 5-methylcytosine, N4methylcytosine and N6-methyladenine in either DNA or $\mathrm{RNA}^{33,60,61}$ as well as in protein glutamine methylation ${ }^{62}$. The phenylalanine (or tyrosine) of motif I provides an edge-to-face interaction to the face of SAM-adenosyl ring, helping to hold it in place. However, in CamA, motif I (SxGxG) has replaced the corresponding phenylalanine with a much smaller Ser62 (Fig. 6b), which interacts with ribose $\mathrm{O}^{\prime}$ oxygen (Fig. 5i). This version of motif I is fully conserved among examined CamA orthologs (Supplementary Fig. 7) and, in fact, is a general feature of the $\gamma$ class of DNA MTases ${ }^{33}$. We now know that the phenylalanine's role can be provided from Phe 200 of helix $\alpha \mathrm{H}$ (see Fig. 5i for the spatial relationship between Ser62 and Phe200). We note that TaqI MTase too has a motif I (AxAxG) with a small-residue substitution for phenylalanine in the corresponding position. Similarly, the SAM-interacting phenylalanine in M.TaqI is Phe146 32 ; the corresponding Phe200 of CamA is very highly conserved among CamA orthologs (Supplementary Fig. 7). Nevertheless, unlike CamA, M.TaqI has an affinity for SAM in the normal range, with a $K_{\mathrm{D}}$ value of $2 \mu \mathrm{M}^{32}$. We note that another MTase, M.EcoP15I (which has a consensus FxGxG motif), has been crystallized in a complex with DNA, but the cofactor is absent ${ }^{44}$.

\section{Discussion}

Here we describe the interaction of $C$. difficile CamA with its cognate DNA substrate, resulting in a rearranged A5:T6 base pair 
immediately $5^{\prime}$ to the flipped-out target adenine (A6). The shifted A5:T6 base pair stacks against the $3^{\prime}$ G7:C7 base pair, as if the target A6 was being squeezed out. To be clear, the temporal sequence of events has not yet been determined-whether the "squeezing" precedes and triggers the base flipping, or follows it and prevents the target adenine from flipping back into the double helix is unknown. We note that a shifted base pair has previously been observed for M.HaeIII ${ }^{63}$, a DNA cytosine MTase, which methylates the internal cytosine (C3) of GGCC. The outer $3^{\prime}$ cytosine (C4) is moved over to form a rearranged base pair with the guanine (G3) that was originally paired with the flippedout target cytosine. However, there is no apparent base pair stacking with the rearranged C4:G3 base pair in available crystal structures, and thus there is no evidence for "squeezing" as seen in CamA.

We also performed enzymatic analysis of CamA to establish the kinetic parameters, seeking to understand the molecular details underlying CamA catalysis coupled with structural analysis. The observation of weak binding of SAM, together with cofactor-induced conformational change of $\mathrm{N}$-terminal residues near the SAM binding pocket, might provide avenues for inhibiting CamA activity using SAM analogs ${ }^{64-66}$. Iterative cycles of crystallography, synthesis, and bioactivity assays would aid successful design of selective and potent inhibitors of CamAmediated DNA adenine methylation in C. difficile. Such inhibitors could be clinically useful, given that CamA-mediated methylation is required for normal sporulation and colonization by C. difficile ${ }^{24,67}$. The profound conformational change when CamA has or lacks a bound cofactor is relatively unique. Further study including mutagenesis is needed to address the mechanism CamA uses for the bi-substrate reaction, and to determine the rate limiting step of the methylation reaction, though some MTases (including phage T4 Dam) are known for which SAH release is the rate-limiting $\operatorname{step}^{68}$. It is also possible that the unusually low affinity of CamA for SAM makes it particularly sensitive to in vivo SAM levels, which could play a regulatory role, as has been suggested for MettL16 $6^{69,70}$.

Is there epigenetic regulation of gene expression in $C$ difficile? It would be useful to understand in greater depth the molecular basis for the CamA requirement for $C$. difficile sporulation and colonization, though the defect in a $\triangle c a m A$ strain appears to be associated with promoters responsive to sigma factors $\mathrm{E}$ and $\mathrm{F}$, and the CAAAAA target overlaps with key regulators such as $\mathrm{CodY}^{24,71}$. Some other bacterial orphan MTases (not associated with a restriction endonuclease) are involved in chromosome replication, DNA repair, and epigenetic gene regulation ${ }^{16,23}$. For example, the expression of pyelonephritis-associated pili (Pap) in uropathogenic E. coli is epigenetically controlled by the methylation state of the two GATC sites (separated by $\sim 90$ nucleotides) in the Pap regulatory region ${ }^{72,73}$. In contrast to most GATC sites in the E. coli genome, the pap-associated sites are not always completely re-methylated after DNA replication, and their methylation state determines in part the phase variation of pilus formation ${ }^{73}$. The failure to methylate these sites is due in part to the binding of regulatory proteins (Lrp, PapI) that block access of $\operatorname{Dam}^{74-76}$.

If CamA is playing a role in epigenetic gene regulation, one would expect to find examples of unmethylated target sites associated with specific gene regulatory regions. In the 36 SMRTsequenced $C$. difficile DNA methylomes, there are an average of 7721 occurrences of the CamA recognition sequence, and among them an average of $21.5(0.3 \%)$ CAAAAA sites per genome are unmethylated in DNA isolated from log-phase cells ${ }^{24}$. The range varied from 33 genomes containing $\sim 10-30$ non-methylated sites and one genome each at 36, 54, and 152 unmethylated sites (supplementary Table S7A of ref. ${ }^{24}$ ). Some of these are conserved sites that are unmethylated in a majority of the 36 genomes.

Finally, the role of CamA may help to predict additional features of its kinetic behavior. If the primary role of CamA methylation is regulatory, one would not necessarily expect it to be highly processive, but maybe (like Dam in the control of the Pap locus) responsive to the presence of other regulatory proteins. If, on the other hand, CamA is meant to link the timing of gene expression to replication, as in the case of some $E$. coli transposons responsive to Dam methylation ${ }^{77-81}$, it might act more processively on just the daughter strand by following the replication fork, in which case only CAAAAA sites on the leading strand would likely be relevant. In this regard, it is interesting that the presence or absence of sinefungin (a SAM analog) or SAH has little effect $(<2 \times)$ on CamA's DNA affinity (Fig. 1e). This is consistent with a possible processive mechanism, where SAH-SAM exchange occurs without dissociation from the DNA, though this remains to be tested.

In summary, while it remains to be fully explained why CamA is essential for normal sporulation and gastrointestinal tract colonization by the major pathogen $C$. difficile, it is now clear that this DNA methyltransferase has unusual features that may help in making it a therapeutic target.

\section{Methods}

CamA gene expression and protein purification. C. difficile 630 strain CamA full length cDNA (gene CD630_27580) was synthesized by Genscript and was cloned into a modified pET-28b vector with a N-terminal His-Sumo tag using a NdeI/ BamHI site for creating the expression construct (pXC2184), which was transformed into an Escherichia coli Rosetta strain. His-Sumo tagged CamA full length protein was expressed with autoinduction medium ${ }^{82}$. Briefly, an overnight culture grown in $3 \mathrm{~mL}$ MDAG medium was inoculated to $3 \mathrm{~L} \mathrm{ZYM-5052} \mathrm{medium} \mathrm{and}$ shaken at $37^{\circ} \mathrm{C}$ until the $\mathrm{OD}_{600 \mathrm{~nm}}$ reached 0.8 . The temperature was then adjusted to $22^{\circ} \mathrm{C}$ and cells were cultured overnight to allow autoinduction of target protein. Cells were harvested, resuspended and lysed by sonification in lysis buffer $500 \mathrm{mM}$ $\mathrm{NaCl}, 20 \mathrm{mM}$ imidazole, $20 \mathrm{mM}$ Tris- $\mathrm{HCl} \mathrm{pH} 8.0$, 5\% glycerol, $0.5 \mathrm{mM}$ tris(2carboxyethyl)phosphine (TCEP) supplied with $0.1 \mathrm{mM}$ phenylmethylsulfonyl fluoride (PMSF). The clarified supernatant containing His-sumo tagged CamA was then loaded onto a 5-mL HisTrap ${ }^{\mathrm{TM}} \mathrm{HP}$ column (GE healthcare), and target CamA protein was eluted with a linear gradient of 20-500 mM imidazole. Fractions containing target protein were pooled together and cut with ULP protease at $4{ }^{\circ} \mathrm{C}$ overnight, leaving two additional $\mathrm{N}$-terminal residues (His-Met). The cleaved protein was diluted three times with lysis buffer without $\mathrm{NaCl}$ and imidazole and loaded onto a tandem HiTrap Q-SP column (GE healthcare $)^{83}$. After sample was loaded, the Q column was removed, and target protein bound in SP column was eluted with a linear gradient of $0.1-1 \mathrm{M} \mathrm{NaCl}$. Fractions with target protein were pooled and further purified on HiLoad Superdex 200 16/60 (GE healthcare), in buffer $300 \mathrm{mM} \mathrm{NaCl}, 20 \mathrm{mM}$ Tris- $\mathrm{HCl}$ pH 7.5, $0.5 \mathrm{mM}$ TCEP. Fractions with high purity of target protein (judged by SDS PAGE; Supplementary Fig. 1a) were pooled, concentrated to $2.5 \mathrm{mg} / \mathrm{mL}$, flash-frozen in liquid nitrogen and stored in $-80^{\circ} \mathrm{C}$.

For expression of L-selenomethionine-substituted CamA, $2.5 \mathrm{~mL}$ of an overnight culture in MDAG medium was inoculated into $250 \mathrm{~mL}$ adaptable medium containing $0.2 \times \mathrm{LB}, 0.8 \times \mathrm{M} 9,5 \%$ glucose, and cultured at $37^{\circ} \mathrm{C}$ until $\mathrm{OD}_{600 \mathrm{~nm}}$ reached 1.0. Cells were centrifuged, resuspended with $25 \mathrm{~mL}$ expression medium containing $1 \times$ M9, 0.65\% Yeast Nitrogen Base (BD 233520), 5\% glucose. A $5-\mathrm{mL}$ of the resuspended culture was inoculated to $0.5 \mathrm{~L}$ expression medium and grown in $37^{\circ} \mathrm{C}$ until the $\mathrm{OD}_{600 \mathrm{~nm}}$ reached to 0.8 . Temperature was shifted to $18^{\circ} \mathrm{C}$ and individual amino acids were added (L-SeMet at $30 \mathrm{mg}$, Lys, Thr, and Phe at 50 $\mathrm{mg}$, Leu, Ile, and Val at $25 \mathrm{mg}$ ). Fifteen min later, target protein was induced with $0.2 \mathrm{mM}$ isopropyl $\beta$-D-1-thiogalactopyranoside (IPTG) at $18^{\circ} \mathrm{C}$ for $15 \mathrm{~h}$. Purification of SeMet-substituted CamA was the same as the wild type.

SAM dependent methylation assay. The DNA methylation activity of CamA was measured by Promega luminescence assay (MTase-Glo $\left.{ }^{\mathrm{TM}}\right)^{84}$, in which the produced SAH product was converted into ATP in a two-step reaction and the ATP was detected by a luciferase reaction. MTase-Glo luminescence assay produces lower positive false signal compared to other methods ${ }^{85}$ and has been employed in our recent studies on SAM-dependent methylation assays ${ }^{70,86-89}$. Typically, for a $10 \mu \mathrm{L}$ reaction, $2 \times(\mathrm{CamA}$ and $\mathrm{SAM})$ was preincubated at room temperature $\left(\sim 22^{\circ} \mathrm{C}\right)$ and the reaction was started by adding the same volume of $2 \times$ DNA substrate (5'-CGA TTC AAA AAG TCC CAA G-3' and $3^{\prime}$-GCT AAG TTT TTC AGG GTT C-5'). Reactions were terminated by adding trifluoroacetic acid (TFA) to 0.1 or $0.2 \%$ final concentration. The $5 \mu \mathrm{L}$ of the reaction mixture was added to 
low-volume 384-well plate and the luminescence signal was measured by a Synergy 4 multimode microplate reader (BioTek). In reaction buffer containing $20 \mathrm{mM}$ Tris-HCl, $0.1 \%$ TFA was used or $0.2 \%$ TFA for reaction buffer with $100 \mathrm{mM}$ Tris$\mathrm{HCl}$ to stop the reaction.

For variation of $\mathrm{pH}$ (Supplementary Fig. 1c), we used two buffer systems in $50 \mathrm{mM} \mathrm{NaCl}, 0.1 \mathrm{mg} / \mathrm{mL}$ BSA and $1 \mathrm{mM}$ DTT: (1) $10 \mathrm{mM}$ citric acid and $10 \mathrm{mM}$ bis-tris propane (CBTP) for $\mathrm{pH} 5.5$ to 8.8 , (2) $20 \mathrm{mM}$ Tris- $\mathrm{HCl}$ for $\mathrm{pH} 7.0$ to 8.4 , with CamA (50 nM or $100 \mathrm{nM}$ ), $20 \mu \mathrm{M}$ DNA, and $30 \mu \mathrm{M}$ SAM. Reactions lasted for $10 \mathrm{~min}$.

For variation of ionic strength (Supplementary Fig. 1d), $\mathrm{NaCl}$ concentration was varied from 0 to $175 \mathrm{mM} \mathrm{NaCl}$ in $20 \mathrm{mM}$ Tris- $\mathrm{HCl} \mathrm{pH} \mathrm{7.5,} 0.1 \mathrm{mg} / \mathrm{mL}$ BSA, $1 \mathrm{mM}$ DTT, $50 \mathrm{nM}$ CamA, $20 \mu \mathrm{M}$ DNA, and $30 \mu \mathrm{M}$ SAM. Reactions lasted for $10 \mathrm{~min}$

For variation of the reaction time (Supplementary Fig. 1e), the reaction was carried out with varying time in the optimal buffer $(100 \mathrm{mM} \mathrm{NaCl}, 20 \mathrm{mM}$ Tris-HCl pH 7.5, $0.1 \mathrm{mg} / \mathrm{mL}$ BSA, $1 \mathrm{mM}$ DTT) with $25 \mathrm{nM} \mathrm{CamA,} 20 \mu \mathrm{M}$ DNA, and $50 \mu \mathrm{M}$ SAM.

For variation of the enzyme concentration (Supplementary Fig. 1f), reactions were conducted in the optimal buffer with $20 \mu \mathrm{M}$ DNA and $30 \mu \mathrm{M}$ SAM for $2.5 \mathrm{~min}$.

To measure the $K_{\mathrm{m}}$ for SAM (Fig. 1a), the reactions were carried out with $50 \mathrm{nM}$ CamA, $20 \mu \mathrm{M}$ DNA with varying SAM concentration for $2.5 \mathrm{~min}$ in the optimal buffer. To measure the $K_{\mathrm{m}}$ for DNA (Fig. lb), the reactions were $10 \mathrm{nM}$ CamA, $100 \mu \mathrm{M}$ SAM $\left(\sim 5 \times\right.$ above the $K_{\mathrm{m}}$ value for SAM) with varying DNA concentration in the optimized buffer containing $100 \mathrm{mM}$ Tric-HCl for $2.5 \mathrm{~min}$. We note that $100 \mu \mathrm{M}$ SAM changes the $\mathrm{pH}$ of the mixture and $100 \mathrm{mM}$ Tris- $\mathrm{HCl}$ is necessary to maintain the $\mathrm{pH}$ value around 7.5.

Under the single turnover conditions (Fig. 3h), reactions were carried out overnight with $500 \mathrm{nM}$ CamA, $30 \mu \mathrm{M}$ SAM, and varying DNA concentration at 125,250 , and $500 \mathrm{nM}$ in the optimized buffer.

For DNA oligos with base pair substitutions (Fig. 3i), reactions were conducted with $50 \mathrm{nM}$ CamA, $5 \mu \mathrm{M}$ DNA, $40 \mu \mathrm{M}$ SAM in the optimized buffer for $2.5 \mathrm{~min}$.

Isothermal titration calorimetry. All ITC experiments were performed with a MicroCal PEAQ-ITC automated system (Malvern) at $25^{\circ} \mathrm{C}$ with reference power of $8 \mu \mathrm{cal} / \mathrm{s}$. Nineteen injections were performed with an initial injection of $0.2 \mu \mathrm{L}$ followed by eighteen injections (each of $2 \mu \mathrm{L}$ ) with continuous stirring at $750 \mathrm{rpm}$. The duration time for the first injection was set at $0.4 \mathrm{~s}$ and fixed at $4 \mathrm{~s}$ for the following injections and the spacing time between injections was set at $300 \mathrm{~s}$ to allow equilibrium.

To measure the binding of CamA to cofactor, SAM, SAH, or sinefungin ( 450 or $900 \mu \mathrm{M})$ was titrated to CamA ( 40 or $45 \mu \mathrm{M})$ in $20 \mathrm{mM}$ Tris- $\mathrm{HCl} \mathrm{pH} \mathrm{7.5,250} \mathrm{mM}$ $\mathrm{NaCl}, 0.5 \mathrm{mM}$ TCEP supplied with $0.9 \%$ or $1.8 \%$ DMSO (Supplementary Fig. 2). For measuring the binding of CamA to DNA, $200 \mu \mathrm{M}$ DNA was titrated to $20 \mu \mathrm{M}$ CamA in $20 \mathrm{mM}$ HEPES pH 8.0, $0.5 \mathrm{mM}$ TCEP, $5 \%$ glycerol, $150 \mathrm{mM} \mathrm{NaCl}$, or $250 \mathrm{mM} \mathrm{NaCl}$ (Supplementary Fig. 3a). To analyze the effect of presence of cofactor on CamA binding to DNA, $200 \mu \mathrm{M}$ DNA was titrated to $18 \mu \mathrm{M}$ CamA with or without $100 \mu \mathrm{M}$ SAH or sinefungin (Supplementary Fig. 3b). For all ITC experiments, cofactor or DNA oligos in the syringe was titrated to the corresponding buffer as a control and no heat of dilution was detected. The binding data were fitted as "one site" and binding constants were calculated using the ITC analysis module supplied by the manufacturer.

Purification of protein-DNA complexes for crystallization. Two complementary DNA oligonucleotides (5'-TTC AAA AAG TCC CA-3' and $3^{\prime}$ - AGT TTT TCA GGG TA-5') were annealed with $2 \mathrm{mM}$ concentration in $20 \mathrm{mM}$ Tris-HCl pH 7.5, $50 \mathrm{mM} \mathrm{NaCl}$. SAH in stock solution of $25 \mathrm{mM}$ were dissolved in $0.4 \% \mathrm{HCl}$. Purified CamA, dsDNA oligo, and SAH were initially mixed in $2 \mathrm{~mL}$ at molar ratio 1:1.1:10 $(12.5 \mu \mathrm{M}$ [E], 13.8 $\mu \mathrm{M}$ [DNA], and $125 \mu \mathrm{M}$ [SAH] $)$ in buffer of $150 \mathrm{mM}$ $\mathrm{NaCl}, 20 \mathrm{mM}$ Tris-HCl pH 7.5, $0.5 \mathrm{mM}$ TCEP and incubated on ice for $2 \mathrm{~h}$. The 2$\mathrm{mL}$ complex was concentrated by $4 \times$ to $\sim 0.5 \mathrm{~mL}$ and loaded onto a Superdex 200 Increase 10/300 GL column (GE healthcare) in buffer of $100 \mathrm{mM} \mathrm{NaCl}, 20 \mathrm{mM}$ Tris- $\mathrm{HCl} \mathrm{pH} 7.5$ and $0.5 \mathrm{mM}$ TCEP. Extra DNA molecules were separated and fractions with CamA-DNA complex (Supplementary Fig. 4a) were pooled and concentrated to about $80 \mu \mathrm{M}(\sim 5.2 \mathrm{mg} / \mathrm{mL})$. We note that although SAH was used in the initial complex mixture, the cofactor did not carry through the column.

To prepare the ternary complex of CamA, DNA and SAH, the three components were mixed at concentration of $12.5 \mu \mathrm{M}[\mathrm{CamA}], 14.4 \mu \mathrm{M}[\mathrm{DNA}]$ and $300 \mu \mathrm{M}$ [SAH] in $1.5 \mathrm{~mL}$ of $100 \mathrm{mM} \mathrm{NaCl}, 20 \mathrm{mM}$ Tris-HCl pH 7.5, $0.5 \mathrm{mM}$ TCEP, and incubated on ice for $2 \mathrm{~h}$. The ternary complex was concentrated about $8 \times$ to a final concentration of about $100 \mu \mathrm{M}$ [CamA], $115 \mu \mathrm{M}$ [DNA], and $300 \mu \mathrm{M}$ $[\mathrm{SAH}]$ and directly used for crystallization.

Crystallization was carried out by an Art Robbins Gryphon Crystallization Robot. Mixture of $0.2 \mu \mathrm{L}$ complex with $0.2 \mu \mathrm{L}$ crystallization solution over $70 \mu \mathrm{L}$ well solution was set up using the sitting drop technique. Single crystals were obtained from solution containing $0.1 \mathrm{M}$ Bis-Tris $\mathrm{pH} 6.7,23 \%$ polyethylene glycol (PEG) 3350 and $0.3 \mathrm{M}$ potassium citrate. Crystals of SeMet-CamA and DNA complex (without added cofactor) grew in solution containing $0.1 \mathrm{M}$ Tris- $\mathrm{HCl} \mathrm{pH}$ 7.0, 24.5\% PEG 3350 and $0.28 \mathrm{M}$ potassium citrate (Supplementary Fig. $4 \mathrm{~d}$ ). Similarly, the ternary complex of CamA, DNA and SAH were crystallized under conditions of $0.1 \mathrm{M}$ Tris- $\mathrm{HCl} \mathrm{pH} \mathrm{7.3,23 \%} \mathrm{PEG} \mathrm{3350,} \mathrm{and} 0.27 \mathrm{M}$ potassium citrate.
X-ray crystallography. When trays with crystallization drops were opened to obtain crystals, some phase separation occurred in the drop and over a short period of time, crystals would tend to dissolve. Thus, crystals were picked up quickly in a nylon loop which were then momentarily placed into mother liquor supplemented with $20 \%(\mathrm{v} / \mathrm{v})$ ethylene glycol before plunging into liquid nitrogen for cryoprotection. X-ray diffraction data were collected at the SER-CAT beamline 22ID of the Advanced Photon Source at Argonne National Laboratory. For the crystal with the selenomethionyl CamA, X-ray diffraction data was collected $15 \mathrm{eV}$ above the selenium absorption edge $(0.97775 \AA)$ and 1000 images were collected rotating the crystal $1^{\circ}$ per image so as to obtain large redundancy to achieve an adequate anomalous signal.

The resultant dataset was examined using the Xtriage module of PHENIX ${ }^{90}$ which reported a very good anomalous signal to $5.1 \AA$ resolution. The AutoSol module of PHENIX ${ }^{11}$ found 15 selenium atoms (five for each protein; Supplementary Fig. 4c) with a Figure-Of-Merit of 0.47 and gave a density-modified map with an R-factor of 0.27 . The initial electron density showed recognizable features of secondary structures of $\beta$-sheets and $\alpha$-helices. Reinserting the selenium positions into AutoSol and utilizing the full resolution of the dataset $(2.69 \AA)$ gave a very good map in which protein side chains and DNA could be easily identified. In this instance, Autosol reported a Figure-Of-Merit of 0.24 and gave a densitymodified map with an R-factor of 0.24 . AutoBuild module of PHENIX was utilized to begin the model building which allowed for reiterative processing, and together with manual building in COOT $^{92}$, giving improved maps which allowed building of three protein-DNA complexes in the asymmetric unit. COOT was also utilized for corrections between PHENIX refinement rounds.

Data for native CamA crystals were collected at wavelength of $1.00000 \AA$. The native structures with and without SAH were solved by the difference Fourier method (Supplementary Table 1). In the structure with SAH, difference electron density of bound SAH and the additional residues at the N-termini in the asymmetric unit were immediately obvious and easily built using COOT before refinement. Structure quality was analyzed during PHENIX refinements and finally validated by the PDB validation server ${ }^{93}$. Molecular graphics were generated by using PyMol (Schrödinger, LLC)

Reporting summary. Further information on research design is available in the Nature Research Reporting Summary linked to this article.

\section{Data availability}

The experimental data that support the findings of this study are contained within the article. The X-ray structure (coordinates) and the source data (structure factor file) of CamA with bound DNA have been submitted to the PDB under accession numbers 7LNI (SeMet-CamA+DNA), 7LNJ (CamA + DNA) and 7LT5 (CamA + DNA + SAH). The source data underlying Figs. 1a, 1b, 3h, 31 and Supplementary Fig. 1 are provided as a Source Data file with this paper. Source data are provided with this paper.

Received: 8 February 2021; Accepted: 13 May 2021; Published online: 08 June 2021

\section{References}

1. Smits, W. K., Lyras, D., Lacy, D. B., Wilcox, M. H. \& Kuijper, E. J. Clostridium difficile infection. Nat. Rev. Dis. Prim. 2, 16020 (2016).

2. Guh, A. Y. \& Kutty, P. K. Clostridioides difficile Infection. Ann. Intern. Med. 169, ITC49-ITC64 (2018)

3. Turner, N. A., Smith, B. A. \& Lewis, S. S. Novel and emerging sources of Clostridioides difficile infection. PLoS Pathog. 15, e1008125 (2019).

4. Webb, B. J. et al. Antibiotic exposure and risk for hospital-associated Clostridioides difficile infection. Antimicrob. Agents Chemother. 64, e02169-19 (2020).

5. Rashid, T. et al. Activity of hospital disinfectants against vegetative cells and spores of Clostridioides difficile embedded in biofilms. Antimicrob. Agents Chemother. 64, e01031-19 (2019).

6. Freedberg, D. E., Salmasian, H., Cohen, B., Abrams, J. A. \& Larson, E. L. Receipt of antibiotics in hospitalized patients and risk for Clostridium difficile infection in subsequent patients who occupy the same bed. JAMA Intern. Med. 176, 1801-1808 (2016).

7. Markham, N. O. et al. Murine intrarectal instillation of purified recombinant C. difficile toxins enables mechanistic studies of pathogenesis. Infect. Immun. 89, e00543-20 (2021).

8. Skinner, A. M. et al. The relative role of toxins A and B in the virulence of Clotridioides difficile. J. Clin. Med. 10, 96 (2020).

9. Stewart, D., Anwar, F. \& Vedantam, G. Anti-virulence strategies for Clostridioides difficile infection: advances and roadblocks. Gut Microbes 12, 1802865 (2020).

10. Kachrimanidou, M. \& Tsintarakis, E. Insights into the role of human gut microbiota in Clostridioides difficile infection. Microorganisms 8, 200 (2020). 
11. Lessa, F. C. et al. Burden of Clostridium difficile infection in the United States. N. Engl. J. Med. 372, 825-834 (2015).

12. Gil, F., Calderon, I. L., Fuentes, J. A. \& Paredes-Sabja, D. Clostridioides (Clostridium) difficile infection: current and alternative therapeutic strategies. Future Microbiol. 13, 469-482 (2018).

13. Orenstein, R., Patron, R. L. \& Seville, M. T. Why does Clostridium difficile infection recur? J. Am. Osteopath. Assoc. 119, 322-326 (2019).

14. Gupta, A., Saha, S. \& Khanna, S. Therapies to modulate gut microbiota: past, present and future. World J. Gastroenterol. 26, 777-788 (2020).

15. Mondal, S. I., Draper, L. A., Ross, R. P. \& Hill, C. Bacteriophage endolysins as a potential weapon to combat Clostridioides difficile infection. Gut Microbes 12, 1813533 (2020).

16. Nye, T. M., Fernandez, N. L. \& Simmons, L. A. A positive perspective on DNA methylation: regulatory functions of DNA methylation outside of host defense in Gram-positive bacteria. Crit. Rev. Biochem. Mol. Biol. 55, 576-591 (2020).

17. Sanchez-Romero, M. A. \& Casadesus, J. The bacterial epigenome. Nat. Rev. Microbiol. 18, 7-20 (2020).

18. Deng, X. et al. Widespread occurrence of N6-methyladenosine in bacterial mRNA. Nucleic Acids Res. 43, 6557-6567 (2015).

19. Nye, T. M. et al. Methyltransferase DnmA is responsible for genome-wide N6methyladenosine modifications at non-palindromic recognition sites in Bacillus subtilis. Nucleic Acids Res. 48, 5332-5348 (2020).

20. Wyrzykowski, J. \& Volkert, M. R. The Escherichia coli methyl-directed mismatch repair system repairs base pairs containing oxidative lesions. J. Bacteriol. 185, 1701-1704 (2003).

21. Stephens, C., Reisenauer, A., Wright, R. \& Shapiro, L. A cell cycle-regulated bacterial DNA methyltransferase is essential for viability. Proc. Natl Acad. Sci. USA 93, 1210-1214 (1996)

22. Roberts, R. J., Vincze, T., Posfai, J. \& Macelis, D. REBASE-a database for DNA restriction and modification: enzymes, genes and genomes. Nucleic Acids Res. 43, D298-D299 (2015).

23. Marinus, M. G. \& Casadesus, J. Roles of DNA adenine methylation in hostpathogen interactions: mismatch repair, transcriptional regulation, and more. FEMS Microbiol. Rev. 33, 488-503 (2009).

24. Oliveira, P. H. et al. Epigenomic characterization of Clostridioides difficile finds a conserved DNA methyltransferase that mediates sporulation and pathogenesis. Nat. Microbiol. 5, 166-180 (2020).

25. Roberts, A. P. \& Smits, W. K. The evolving epidemic of Clostridium difficile 630. Anaerobe 53, 2-4 (2018).

26. Urig, S. et al. The Escherichia coli dam DNA methyltransferase modifies DNA in a highly processive reaction. J. Mol. Biol. 319, 1085-1096 (2002).

27. Woodcock, C. B., Yakubov, A. B. \& Reich, N. O. Caulobacter crescentus cell cycle-regulated DNA methyltransferase uses a novel mechanism for substrate recognition. Biochemistry 56, 3913-3922 (2017).

28. Bergerat, A. \& Guschlbauer, W. The double role of methyl donor and allosteric effector of S-adenosyl-methionine for Dam methylase of E. coli. Nucleic Acids Res. 18, 4369-4375 (1990).

29. Marzabal, S. et al. Dam methylase from Escherichia coli: kinetic studies using modified DNA oligomers: hemimethylated substrates. Nucleic Acids Res. 23, 3648-3655 (1995).

30. Zinoviev, V. V. et al. Phage T4 DNA [N6-adenine] methyltransferase: kinetic studies using oligonucleotides containing native or modified recognition sites. Biol. Chem. 379, 481-488 (1998).

31. Posnick, L. M. \& Samson, L. D. Influence of S-adenosylmethionine pool size on spontaneous mutation, dam methylation, and cell growth of Escherichia coli. J. Bacteriol. 181, 6756-6762 (1999).

32. Schluckebier, G., Kozak, M., Bleimling, N., Weinhold, E. \& Saenger, W Differential binding of S-adenosylmethionine S-adenosylhomocysteine and Sinefungin to the adenine-specific DNA methyltransferase M.TaqI. J. Mol. Biol. 265, 56-67 (1997).

33. Malone, T., Blumenthal, R. M. \& Cheng, X. Structure-guided analysis reveals nine sequence motifs conserved among DNA amino-methyltransferases, and suggests a catalytic mechanism for these enzymes. J. Mol. Biol. 253, 618-632 (1995).

34. Hendrickson, W. A., Horton, J. R. \& LeMaster, D. M. Selenomethionyl proteins produced for analysis by multiwavelength anomalous diffraction (MAD): a vehicle for direct determination of three-dimensional structure. Embo J. 9, 1665-1672 (1990)

35. Trautner, T. A., Pawlek, B., Behrens, B. \& Willert, J. Exact size and organization of DNA target-recognizing domains of multispecific DNA(cytosine-C5)-methyltransferases. EMBO J. 15, 1434-1442 (1996).

36. Lauster, R., Trautner, T. A. \& Noyer-Weidner, M. Cytosine-specific type II DNA methyltransferases. A conserved enzyme core with variable targetrecognizing domains. J. Mol. Biol. 206, 305-312 (1989).

37. Schubert, H. L., Blumenthal, R. M. \& Cheng, X. Many paths to methyltransfer: a chronicle of convergence. Trends Biochem. Sci. 28, 329-335 (2003).

38. Cheng, X. Structure and function of DNA methyltransferases. Annu. Rev. Biophys. Biomol. Struct. 24, 293-318 (1995).
39. Cheng, X. DNA modification by methyltransferases. Curr. Opin. Struct. Biol. 5, 4-10 (1995).

40. Labahn, J. et al. Three-dimensional structure of the adenine-specific DNA methyltransferase M.Taq I in complex with the cofactor Sadenosylmethionine. Proc. Natl Acad. Sci. USA 91, 10957-10961 (1994).

41. Schluckebier, G., O'Gara, M., Saenger, W. \& Cheng, X. Universal catalytic domain structure of AdoMet-dependent methyltransferases. J. Mol. Biol. 247, 16-20 (1995).

42. Goedecke, K., Pignot, M., Goody, R. S., Scheidig, A. J. \& Weinhold, E. Structure of the N6-adenine DNA methyltransferase M.TaqI in complex with DNA and a cofactor analog. Nat. Struct. Biol. 8, 121-125 (2001).

43. Klimasauskas, S., Kumar, S., Roberts, R. J. \& Cheng, X. HhaI methyltransferase flips its target base out of the DNA helix. Cell 76, 357-369 (1994).

44. Gupta, Y. K., Chan, S. H., Xu, S. Y. \& Aggarwal, A. K. Structural basis of asymmetric DNA methylation and ATP-triggered long-range diffusion by EcoP15I. Nat. Commun. 6, 7363 (2015).

45. Horton, J. R. et al. The cell cycle-regulated DNA adenine methyltransferase CcrM opens a bubble at its DNA recognition site. Nat. Commun. 10, 4600 (2019).

46. Timar, E., Groma, G., Kiss, A. \& Venetianer, P. Changing the recognition specificity of a DNA-methyltransferase by in vitro evolution. Nucleic Acids Res. 32, 3898-3903 (2004).

47. Luscombe, N. M., Laskowski, R. A. \& Thornton, J. M. Amino acid-base interactions: a three-dimensional analysis of protein-DNA interactions at an atomic level. Nucleic Acids Res. 29, 2860-2874 (2001).

48. Patel, A., Horton, J. R., Wilson, G. G., Zhang, X. \& Cheng, X. Structural basis for human PRDM9 action at recombination hot spots. Genes Dev. 30, 257-265 (2016).

49. Hashimoto, H. et al. Structural basis for the versatile and methylationdependent binding of CTCF to DNA. Mol. Cell 66, 711-720 (2017).

50. Lan, X. et al. ZNF410 uniquely activates the NuRD component CHD4 to silence fetal hemoglobin expression. Mol. Cell 81, 239-254 (2021).

51. Horowitz, S. \& Trievel, R. C. Carbon-oxygen hydrogen bonding in biological structure and function. J. Biol. Chem. 287, 41576-41582 (2012).

52. Patel, A. et al. DNA conformation induces adaptable binding by tandem zinc finger proteins. Cell 173, 221-233 (2018).

53. Messer, W. \& Noyer-Weidner, M. Timing and targeting: the biological functions of Dam methylation in E. coli. Cell 54, 735-737 (1988).

54. Yang, Z. et al. Structure of the bacteriophage T4 DNA adenine methyltransferase. Nat. Struct. Biol. 10, 849-855 (2003)

55. Horton, J. R., Liebert, K., Hattman, S., Jeltsch, A. \& Cheng, X. Transition from nonspecific to specific DNA interactions along the substrate-recognition pathway of dam methyltransferase. Cell 121, 349-361 (2005).

56. Horton, J. R., Liebert, K., Bekes, M., Jeltsch, A. \& Cheng, X. Structure and substrate recognition of the Escherichia coli DNA adenine methyltransferase. J. Mol. Biol. 358, 559-570 (2006).

57. Woodcock, C. B., Horton, J. R., Zhang, X., Blumenthal, R. M. \& Cheng, X. Beta class amino methyltransferases from bacteria to humans: evolution and structural consequences. Nucleic Acids Res. 48, 10034-10044 (2020).

58. Berdis, A. J. et al. A cell cycle-regulated adenine DNA methyltransferase from Caulobacter crescentus processively methylates GANTC sites on hemimethylated DNA. Proc. Natl Acad. Sci. USA 95, 2874-2879 (1998).

59. Chouhan, B. P. S., Maimaiti, S., Gade, M. \& Laurino, P. Rossmann-fold methyltransferases: taking a "beta-Turn" around their cofactor, SAdenosylmethionine. Biochemistry 58, 166-170 (2019).

60. Kumar, S. et al. The DNA (cytosine-5) methyltransferases. Nucleic Acids Res. 22, 1-10 (1994).

61. Bujnicki, J. M., Feder, M., Radlinska, M. \& Blumenthal, R. M. Structure prediction and phylogenetic analysis of a functionally diverse family of proteins homologous to the MT-A70 subunit of the human mRNA:m(6)A methyltransferase. J. Mol. Evol. 55, 431-444 (2002).

62. Bujnicki, J. M. \& Radlinska, M. Is the HemK family of putative Sadenosylmethionine-dependent methyltransferases a "missing" zeta subfamily of adenine methyltransferases? A hypothesis. IUBMB Life 48, 247-249 (1999).

63. Reinisch, K. M., Chen, L., Verdine, G. L. \& Lipscomb, W. N. The crystal structure of HaeIII methyltransferase convalently complexed to DNA: an extrahelical cytosine and rearranged base pairing. Cell 82, 143-153 (1995).

64. Zhang, J. \& Zheng, Y. G. SAM/SAH analogs as versatile tools for SAMdependent methyltransferases. ACS Chem. Biol. 11, 583-597 (2016).

65. Jain, R., Butler, K. V., Coloma, J., Jin, J. \& Aggarwal, A. K. Development of a Sadenosylmethionine analog that intrudes the RNA-cap binding site of Zika methyltransferase. Sci. Rep. 7, 1632 (2017).

66. Ahmed-Belkacem, R. et al. Synthesis of adenine dinucleosides SAM analogs as specific inhibitors of SARS-CoV nsp14 RNA cap guanine-N7methyltransferase. Eur. J. Med. Chem. 201, 112557 (2020).

67. Oliveira, P. H. \& Fang, G. Conserved DNA methyltransferases: a window into fundamental mechanisms of epigenetic regulation in bacteria. Trends Microbiol. 29, 28-40 (2020). 
68. Evdokimov, A. A., Zinoviev, V. V., Malygin, E. G., Schlagman, S. L. \& Hattman, S. Bacteriophage T4 Dam DNA-[N6-adenine]methyltransferase. Kinetic evidence for a catalytically essential conformational change in the ternary complex. J. Biol. Chem. 277, 279-286 (2002).

69. Pendleton, K. E. et al. The U6 snRNA m(6)A methyltransferase METTL16 regulates SAM synthetase intron retention. Cell 169, 824-835 (2017).

70. Yu, D., Kaur, G., Blumenthal, R. M., Zhang, X. \& Cheng, X. Enzymatic characterization of three human RNA adenosine methyltransferases reveals diverse substrate affinities and reaction optima. J. Biol. Chem. 296, 100270 (2021).

71. Brinsmade, S. R. CodY, a master integrator of metabolism and virulence in Gram-positive bacteria. Curr. Genet. 63, 417-425 (2017).

72. Hernday, A., Krabbe, M., Braaten, B. \& Low, D. Self-perpetuating epigenetic pili switches in bacteria. Proc. Natl Acad. Sci. USA 99, 16470-16476 (2002).

73. Hernday, A. D., Braaten, B. A. \& Low, D. A. The mechanism by which DNA adenine methylase and PapI activate the pap epigenetic switch. Mol. Cell 12, 947-957 (2003).

74. Peterson, S. N. \& Reich, N. O. Competitive Lrp and Dam assembly at the pap regulatory region: implications for mechanisms of epigenetic regulation. $J$. Mol. Biol. 383, 92-105 (2008).

75. Kawamura, T., Vartanian, A. S., Zhou, H. \& Dahlquist, F. W. The design involved in PapI and Lrp regulation of the pap operon. J. Mol. Biol. 409, 311-332 (2011).

76. Zamora, M., Ziegler, C. A., Freddolino, P. L. \& Wolfe, A. J. A thermosensitive, phase-variable epigenetic switch: pap revisited. Microbiol. Mol. Biol. Rev. 84, e00030-17 (2020).

77. Morisato, D. \& Kleckner, N. Tn10 transposition and circle formation in vitro. Cell 51, 101-111 (1987).

78. McCommas, S. A. \& Syvanen, M. Temporal control of transposition in Tn5. J. Bacteriol. 170, 889-894 (1988).

79. Makris, J. C., Nordmann, P. L. \& Reznikoff, W. S. Mutational analysis of insertion sequence 50 (IS50) and transposon 5 (Tn5) ends. Proc. Natl Acad. Sci. USA 85, 2224-2228 (1988).

80. Dodson, K. W. \& Berg, D. E. Factors affecting transposition activity of IS50 and Tn5 ends. Gene 76, 207-213 (1989).

81. Tomcsanyi, T. \& Berg, D. E. Transposition effect of adenine (Dam) methylation on activity of O end mutants of IS50. J. Mol. Biol. 209, 191-193 (1989).

82. Studier, F. W. Protein production by auto-induction in high density shaking cultures. Protein Expr. Purif. 41, 207-234 (2005).

83. Patel, A., Hashimoto, H., Zhang, X. \& Cheng, X. Characterization of how DNA modifications affect DNA binding by $\mathrm{C} 2 \mathrm{H} 2$ zinc finger proteins. Methods Enzymol. 573, 387-401 (2016).

84. Hsiao, K., Zegzouti, H. \& Goueli, S. A. Methyltransferase-Glo: a universal, bioluminescent and homogenous assay for monitoring all classes of methyltransferases. Epigenomics 8, 321-339 (2016).

85. Dong, G. et al. Optimization of high-throughput methyltransferase assays for the discovery of small molecule inhibitors. ACS Comb. Sci. 22, 422-432 (2020).

86. Dai, S. et al. Structural basis for the target specificity of actin histidine methyltransferase SETD3. Nat. Commun. 10, 3541 (2019).

87. Dai, S. et al. Characterization of SETD3 methyltransferase-mediated protein methionine methylation. J. Biol. Chem. 295, 10901-10910 (2020).

88. Dai, S. et al. An engineered variant of SETD3 methyltransferase alters target specificity from histidine to lysine methylation. J. Biol. Chem. 295, 2582-2589 (2020).

89. Zeng, Y. et al. The inactive Dnmt3b3 isoform preferentially enhances Dnmt3b-mediated DNA methylation. Genes Dev. 34, 1546-1558 (2020).
90. Adams, P. D. et al. PHENIX: a comprehensive Python-based system for macromolecular structure solution. Acta Crystallogr D Biol. Crystallogr 66 213-221 (2010).

91. Terwilliger, T. C. et al. Decision-making in structure solution using Bayesian estimates of map quality: the PHENIX AutoSol wizard. Acta Crystallogr D Biol. Crystallogr 65, 582-601 (2009).

92. Emsley, P. \& Cowtan, K. Coot: model-building tools for molecular graphics. Acta Crystallogr. D Biol. Crystallogr 60, 2126-2132 (2004).

93. Read, R. J. et al. A new generation of crystallographic validation tools for the protein data bank. Structure 19, 1395-1412 (2011).

\section{Acknowledgements}

We thank Dr. Clayton Woodcock for his initial involvement in CamA activity studies. We thank Ms. Yu Cao for technical assistance. The work was supported by U.S. National Institutes of Health grant R35GM134744 and Cancer Prevention and Research Institute of Texas grant RR160029. X.C. is a CPRIT Scholar in Cancer Research.

\section{Author contributions}

J.R.H. did cloning and initial purifications and crystallizations. J.Z. performed protein purification, enzymatic assays, crystallization and assisted J.R.H. in X-ray crystallography. R.M.B. participated in discussion, performed bioinformatic analysis, and assisted in preparing the manuscript. X.Z. and X.C. organized and designed the scope of the study.

\section{Competing interests}

The authors declare no competing interests.

\section{Additional information}

Supplementary information The online version contains supplementary material available at https://doi.org/10.1038/s41467-021-23693-w.

Correspondence and requests for materials should be addressed to X.Z. or X.C.

Peer review information Nature Communications thanks Yogesh Gupta and the other, anonymous, reviewer(s) for their contribution to the peer review of this work. Peer reviewer reports are available.

Reprints and permission information is available at http://www.nature.com/reprints

Publisher's note Springer Nature remains neutral with regard to jurisdictional claims in published maps and institutional affiliations.

Open Access This article is licensed under a Creative Commons Attribution 4.0 International License, which permits use, sharing, adaptation, distribution and reproduction in any medium or format, as long as you give appropriate credit to the original author(s) and the source, provide a link to the Creative Commons license, and indicate if changes were made. The images or other third party material in this article are included in the article's Creative Commons license, unless indicated otherwise in a credit line to the material. If material is not included in the article's Creative Commons license and your intended use is not permitted by statutory regulation or exceeds the permitted use, you will need to obtain permission directly from the copyright holder. To view a copy of this license, visit http://creativecommons.org/ licenses/by/4.0/.

(C) The Author(s) 2021 